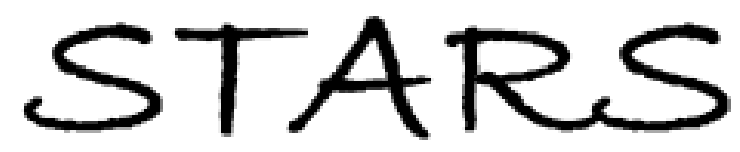

University of Central Florida

STARS

$1-1-2014$

\title{
Quantum corrections to thermopower and conductivity in graphene
}

Aleksander P. Jinz

Stefan Kettemann

Eduardo R. Mucciolo

University of Central Florida

Find similar works at: https://stars.library.ucf.edu/facultybib2010 University of Central Florida Libraries http://library.ucf.edu

This Article is brought to you for free and open access by the Faculty Bibliography at STARS. It has been accepted for inclusion in Faculty Bibliography 2010 s by an authorized administrator of STARS. For more information, please contactSTARS@ucf.edu.

\section{Recommended Citation}

Jinz, Aleksander P.; Kettemann, Stefan; and Mucciolo, Eduardo R., "Quantum corrections to thermopower and conductivity in graphene" (2014). Faculty Bibliography 2010s. 5446.

https://stars.library.ucf.edu/facultybib2010/5446

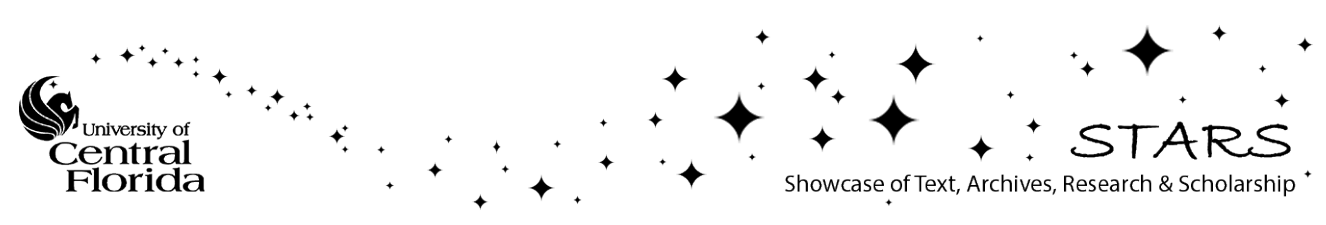




\title{
Quantum corrections to thermopower and conductivity in graphene
}

\author{
Aleksander P. Hinz ${ }^{*}$ and Stefan Kettemann ${ }^{\dagger}$ \\ School of Engineering and Science, Jacobs University Bremen, Bremen 28759, Germany \\ and Asia Pacific Center for Theoretical Physics (APCTP) and Division of Advanced Materials Science, Pohang University of Science \\ and Technology (POSTECH), San31, Hyoja-dong, Nam-gu, Pohang 790-784, South Korea \\ Eduardo R. Mucciolo $\ddagger$ \\ Department of Physics, University of Central Florida, Orlando, Florida 32816-2385, USA
}

(Received 12 April 2013; revised manuscript received 16 January 2014; published 11 February 2014)

\begin{abstract}
The quantum corrections to the conductivity and thermopower in monolayer graphene are studied. We use the recursive Green's-function method to calculate numerically the conductivity and thermopower of graphene. We then analyze these weak-localization corrections by fitting with the analytical theory as a function of the impurity parameters and gate potential. As a result of the quantum corrections to the thermopower, we find large magnetothermopower, which is shown to provide a very sensitive measure of the size and strength of the impurities. We compare these analytical and numerical results with existing experimental measurements of magnetoconductance of single-layer graphene and find that the average size and strength of the impurities in these samples can thereby be determined. We suggest favorable parameter ranges for future measurements of the magnetothermopower.
\end{abstract}

DOI: 10.1103/PhysRevB.89.075411

PACS number(s): 65.80.Ck, 72.20.Pa, 73.22.Pr

\section{INTRODUCTION}

Since its synthesis, graphene [1] has attracted a lot of attention, both for its novel electronic properties and its possible applications. One of the most remarkable aspects is that the weak-localization effect, which is a typical low-temperature phenomenon due to quantum corrections to the conductivity $\delta \sigma$, can be observed up to a temperature range of 200 Kelvin in graphene [2,3]. It has been theoretically predicted that the sign of these corrections strongly depends on the kind of impurities in the graphene sample [4], so that both positive and negative magnetoconductivity, the so-called weak-antilocalization effect, can be observed. This effect is well known to occur due to spin-orbit interaction, even though the latter is typically very weak in graphene. Noting that the graphene lattice is composed of two sublattices, one can formulate the sublattice degree of freedom as an isospin which is strongly coupled to the momentum. Thereby, any elastic scattering that breaks the graphene sublattice symmetry is expected to result in weak antilocalization. This is made more complicated, however, by the fact that there is another discrete degree of freedom in graphene, i.e., the two degenerate Dirac cones, which can correspondingly be formulated by introducing a pseudospin index. Accordingly, any scattering which mixes these two valleys can result in yet another change of the sign of the quantum correction to the conductivity, and thereby to the restoration of weak localization. More recently, thermopower $S$ in graphene has been measured by several groups [5-8]. The classical thermopower of graphene has been calculated with a range of different methods. The experiments show good agreement with the Mott formula, which corresponds to the leading term of the Sommerfeld expansion at low temperatures and large

\footnotetext{
*Corresponding author: a.hinz@jacobs-university.de

†s.kettemann@jacobs-university.de

${ }^{\ddagger}$ mucciolo@physics.ucf.edu
}

gate voltages [9]. Close to the Dirac point, thermopower shows an unusual behavior, being linear in gate voltage, and changing sign. Thus, while usually thermopower is expected to increase as the Fermi energy, electron density, and thereby conductivity are lowered, in graphene the thermopower becomes smaller as the Dirac point is approached and the electron density and conductivity are reduced.

The main aims of this paper are, first, to find out if there are sizable quantum corrections to the thermopower $\delta S$ in graphene and whether they are sensitive to weak magnetic fields and, second, to present and analyze the combined analytical and numerical theory in order to provide the basis for a more quantitative analysis of experimental results on the magnetoconductance of graphene. This will allow one to characterize samples, in particular the density, strength, and size of carrier scatterers, more accurately.

We restrict our attention to the diffusion term of the thermopower and do not consider the phonon drag contribution, which becomes relevant only at high temperatures. In normal metals, the quantum corrections to the diffusion thermopower are known to be dominated by the weaklocalization corrections to the conductivity, yielding $\delta S / S \approx$ $-\delta \sigma / \sigma[10-12]$. Thus, when the conductivity is reduced by the weak-localization correction, the thermopower becomes enhanced. This is expected since the thermopower is known to increase from a metal towards an insulator. Because of the particular electronic properties of graphene, where the sign of the quantum correction can change upon varying the gate voltage, it could be expected that these quantum corrections to thermopower are particularly large in graphene. We therefore determine the strength and the sign of the resulting magnetothermopower and analyze in detail how it changes with impurity parameters such as range, density, and strength, and with gate voltage.

This paper is organized as follows. We start in Sec. II with a short introduction to the electronic properties of graphene. 
We introduce the model for the description of the impurity potential in Sec. III and list the resulting momentum scattering rates and their dependence on the impurity matrix elements. In Sec. IV A, we give a brief review of transport theory, in particular the theory of weak-localization corrections to the conductivity and its application to graphene. In Sec. IV B, we review the theory of thermopower and address leading quantum corrections.

In Sec. V, we present a numerical method to calculate the conductance which is based on the recursive Green's-function technique (Sec. VB). We introduce the Hamiltonian that models impurities in graphene for the numerical calculation in Sec. VC and relate it in Sec. VI to the impurity model introduced in Sec. III. This connection makes it possible to display the related transport scattering rates from Secs. III and IV as functions of the sample parameter of the numerical method (Sec. V), which is done in Sec. VIB.

In Sec. VII, we present the numerical results for the conductance (Sec. VII A) and thermopower (Sec. VII B). In Sec. VIII, we analyze the numerical results by fitting them to the analytical results (Sec. VIII A). In Sec. VIII B, we attempt an ab initio analysis. To this end, we use the relation of the scattering rates to the impurity parameters of the numerical calculations and insert it in the analytical formulas given in Sec. IV. In Sec. VIII C, we present the analytical results for the quantum corrections to the conductance at zero magnetic field. In Sec. VIII D, we present the results for the quantum corrections to the thermopower at zero magnetic field and the resulting magnetic field dependence of the thermopower. In Sec. IX, we compare the numerical and analytical results with experimental results on weak-localization corrections to the conductance. Finally, we draw the conclusions and summarize our results in Sec. X. In Appendix A, the Hamiltonian is given in matrix notation. In Appendix B, we present analytical results for the magnetoconductance when the warping rate is neglected, $1 / \tau_{w}=0$.

\section{ELECTRONIC STRUCTURE OF GRAPHENE}

Let us start with a brief review of the electronic properties of graphene, introducing the notation used in this paper. We follow the convention of McCann and co-workers [4]. For an overview and comparison of notations, see also [13-16].

Graphene is a two-dimensional layer of carbon atoms arranged in a honeycomb lattice. These carbon atoms are connected by strong $\sigma$ bonds with their three neighboring atoms. The corresponding energy bands are filled valence bonds, lying deep below the Fermi energy. The $\pi$ bond leads to the formation of a $\pi$ band, which is exactly half filled in ungated and undoped graphene. Thus, to study transport properties, we can restrict the model to this $\pi$ band. The basis of the Bravais lattice consists of two atoms, with each one forming one sublattice, named A and B sublattice.

The electronic band structure shows two degenerate halffilled cones, which in momentum space are located on the sites of the hexagonal reciprocal lattice. Close to zero energy, i.e., the Dirac points, the dispersion is, in good approximation, linear. Each Dirac cone is part of a sublattice, the $\mathrm{K}$ and $\mathrm{K}^{\prime}$ valleys.
Accordingly, the Hamiltonian can be written as a $4 \times 4$ matrix which, close to the Dirac points in linear approximation, becomes

$$
H_{1}=v_{F} \zeta_{3} \otimes \vec{\sigma} \cdot \vec{k}
$$

where $\zeta_{3}$ is the diagonal Pauli matrix in $\mathrm{K}-\mathrm{K}^{\prime}$ space, $k_{x}$ and $k_{y}$ are components of the momentum $\vec{k}$ in the plane, and

$$
\vec{\sigma}=\left(\begin{array}{l}
\sigma_{1} \\
\sigma_{2}
\end{array}\right) \text {, }
$$

with $\sigma_{1,2}$ the nondiagonal Pauli matrices in the sublattice (A,B) space. The next correction term to the dispersion is quadratic and given by

$$
H_{2}=-\mu\left[\sigma_{1}\left(k_{x}^{2}-k_{y}^{2}\right)-2 \sigma_{2}\left(k_{x} k_{y}\right)\right] \zeta_{0},
$$

where $\mu$ is the parameter of warping, which is given by $[17,18]$

$$
\mu=\frac{3 t a_{0}^{2}}{8} .
$$

In this notation, the four-component Bloch states are given by

$$
\vec{\Psi}^{T}=\left(\Phi_{\mathrm{AK}}, \Phi_{\mathrm{BK}}, \Phi_{\mathrm{BK}^{\prime}}, \Phi_{\mathrm{AK}^{\prime}}\right) .
$$

The Hamiltonian can be more compactly written in the isospin and pseudospin notation, which is particularly convenient for the purpose of understanding the sign of weak-localization corrections to the conductivity. Following the definition of McCann and co-authors [4], we set

$$
\begin{array}{ll}
\Xi_{0}=\zeta_{0} \otimes \sigma_{0}, & \Xi_{1}=\zeta_{3} \otimes \sigma_{1}, \\
\Xi_{2}=\zeta_{3} \otimes \sigma_{2}, & \Xi_{3}=\zeta_{0} \otimes \sigma_{3},
\end{array}
$$

and

$$
\begin{array}{ll}
\Lambda_{0}=\zeta_{0} \otimes \sigma_{0}, & \Lambda_{1}=\zeta_{1} \otimes \sigma_{3}, \\
\Lambda_{2}=\zeta_{2} \otimes \sigma_{3}, & \Lambda_{3}=\zeta_{3} \otimes \sigma_{0},
\end{array}
$$

where $\sigma_{i}, i=1,2,3$, are the Pauli matrices and $\sigma_{0}$ is the identity matrix in the sublattice (A,B) space, and $\zeta_{i}, i=1,2,3$, are the Pauli matrices and $\zeta_{0}$ is the identity matrix in $\mathrm{K}-\mathrm{K}^{\prime}$ space. The vector of the $4 \times 4$ matrices, $\vec{\Xi}^{T}=\left(\Xi_{1}, \Xi_{2}, \Xi_{3}\right)$, is the isospin vector referring to the sublattice $(\mathrm{A}, \mathrm{B})$ degrees of freedom, and $\vec{\Lambda}^{T}=\left(\Lambda_{1}, \Lambda_{2}, \Lambda_{3}\right)$ is the pseudospin vector referring to the valley $\left(\mathrm{K}, \mathrm{K}^{\prime}\right)$ degrees of freedom.

In this notation, the Hamiltonian $H_{1}+H_{2}$ can be written as

$$
\begin{aligned}
H_{0} & =H_{1}+H_{2} \\
& =\underbrace{v_{F} \vec{\Xi} \cdot \vec{k}}_{\text {Dirac cone }}-\underbrace{\mu \Xi_{1}(\vec{\Xi} \cdot \vec{k}) \Lambda_{3} \Xi_{1}(\vec{\Xi} \cdot \vec{k}) \Xi_{1}}_{\text {Warping correction to the Dirac cone }} .
\end{aligned}
$$

Note that $H_{1}$ is independent of the pseudospin $\vec{\Lambda}$, while the warping term $\mathrm{H}_{2}$ breaks the pseudospin symmetry.

\section{IMPURITIES IN GRAPHENE}

Disorder can have many different origins in graphene, such as adatoms and vacancies, while substitutional defects are rather unlikely due to the strength of the $\sigma$ bonding. For the purpose of the transport calculations, it is rather convenient to classify the disorder according to whether it breaks the isospin 
symmetry (A, B sublattices) or the the pseudospin symmetry (K, $\mathrm{K}^{\prime}$ valleys). Thus, the Hamiltonian of nonmagnetic disorder has, in the representation of the pseudospin $\vec{\Lambda}$ and the isospin $\vec{\Xi}$, the general form [4]

$$
H_{\mathrm{imp}}=I V_{0,0}(\vec{r})+\sum_{i, j=1}^{3} \Xi_{i} \Lambda_{j} V_{i, j}(\vec{r}),
$$

where $I$ is the identity matrix. $V_{0,0}(\vec{r})$ is the part of the disorder potential which leaves both the isospin and the pseudospin symmetry invariant, while the other terms are breaking either of these symmetries with the amplitudes $V_{i, j}(\vec{r})$. We note that this disorder Hamiltonian is invariant under time reversal, $\vec{\Lambda} \rightarrow-\vec{\Lambda}$ and $\vec{\Xi} \rightarrow-\vec{\Xi}$, and thus indeed describes nonmagnetic disorder. See Appendix A for the explicit matrix representation of the Hamiltonian.

The corresponding scattering rates in Born approximation are given by

$$
\tau_{i j}^{-1}=\left(\pi v V_{i, j}^{2}\right) / \hbar,
$$

where $v$ is the density of states per spin and valley. We can thus define the total scattering rate as

$$
\tau^{-1}:=\tau_{00}^{-1}+\sum_{i, j=1,2,3} \tau_{i j}^{-1} .
$$

The intervalley scattering rate is obtained by summing over all matrix elements which couple to the transverse pseudospin components $\Lambda_{1}$ and $\Lambda_{2}$, yielding

$$
\tau_{i}^{-1}:=4 \tau_{\perp \perp^{\prime}}^{-1}+2 \tau_{3 \perp}^{-1},
$$

where we introduced $\perp=1,2$ to denote the transverse components, noting that $\tau_{i 1}^{-1}=\tau_{i 2}^{-1}=: \tau_{i \perp}^{-1}$ and $\tau_{1 j}^{-1}=\tau_{2 j}^{-1}=: \tau_{\perp j}^{-1}$, since $V_{1, j}^{2}=V_{2, j}^{2}=: V_{\perp, j}^{2}$ and $V_{i, 1}^{2}=V_{i, 2}^{2}=: V_{i, \perp}^{2}$.

The intravalley scattering rate is accordingly given by

$$
\tau_{z}^{-1}:=2 \tau_{\perp 3}^{-1}+\tau_{33}^{-1} .
$$

The trigonal warping term in the kinetic energy of graphene results in an asymmetry of the dispersion at each valley with respect to momentum inversion [19], while the total Hamiltonian including both valleys preserves that symmetry. Therefore, the pseudospin is expected to precess in the presence of the warping term. In the presence of elastic scattering, the pseudospin channels are expected to relax accordingly, as in motional narrowing, in proportion to the scattering time $\tau$,

$$
\tau_{w}^{-1}=2 \tau\left(\frac{E_{F}^{2} \mu}{\hbar v^{2}}\right)^{2}
$$

\section{TRANSPORT THEORY}

\section{A. Weak localization corrections to the conductance in graphene}

Quantum corrections to the conductance, the so-called weak-localization corrections, originate from the quantum interference of electrons propagating through the sample. On time scales $t$ exceeding the elastic mean free time $\tau$, electrons move diffusively and their return probability can be enhanced due to the constructive interference of the amplitudes for propagation on closed diffusion paths, as long as that time $t$ does not exceed the phase-coherence time $\tau_{\phi}(T)$. In the presence of spin-orbit interaction, the spin precesses as the electron moves on closed paths, and the phase of the amplitudes for clockwise and anticlockwise propagation no longer matches, unless the total spin of these two propagations adds up to zero. This spin singlet channel leads to destructive interference and thereby an enhancement of the conductance, i.e., the weak-antilocalization correction.

Since the spin-orbit interaction in graphene is weak, in the absence of magnetic impurities, there are two independent spin channels in graphene and the quantum corrections are doubled due to this spin degeneracy. However, since the electron momentum $\vec{p}$ is directly coupled to the isospin $\vec{\Xi}$ (due to the A-B sublattice degree of freedom) in the electron Hamiltonian of Eq. (10), any momentum scattering will result in the breaking of the isospin symmetry. Therefore, there is a finite contribution from the interference of two closed electron paths whose total isospin is zero, leading to weak antilocalization in the isospin singlet channel.

Let us now consider the influence of the pseudospin degree of freedom of the two valleys in graphene on the weak-localization corrections. Formulating the conductance corrections in the representation of pseudospin singlet and triplet modes, one has, in two dimensions [4],

$$
\delta \sigma=\frac{2 e^{2} D_{e}}{\pi \hbar} \int \frac{d^{2} Q}{(2 \pi)^{2}}\left(-C_{0}^{0}+C_{0}^{1}+C_{0}^{2}+C_{0}^{3}\right),
$$

where $D_{e}$ is the diffusion constant, which in graphene is related to the elastic-scattering time as $D_{e}=v_{F}^{2} \tau$, and the momentum integral has an upper cutoff $1 / l_{e}$, where $l_{e}=v_{F} \tau$ is the elastic mean free path. The superscript corresponds to the pseudospin and the subscript corresponds to the isospin.

Without magnetic field $(\vec{B}=0)$, the Cooperon modes $C_{0}^{j}$ are given by

$$
C_{0}^{j}(\vec{Q})=\frac{1}{\left(D_{e} \vec{Q}^{2}+\Gamma_{0}^{j}+\tau_{\phi}^{-1}\right)} .
$$

Here, $\tau_{\phi}^{-1}(T)$ is the dephasing rate caused by electron-electron and electron-phonon interactions, which provides the lowenergy cutoff of the diffusion pole of Eq. (18). The elastic scattering from impurities can break the pseudospin symmetry and results in the following pseudospin relaxation rates:

$$
\begin{gathered}
\Gamma_{0}^{0}=0, \\
\Gamma_{0}^{3}=2 \tau_{i}^{-1}, \\
\Gamma_{0}^{1}=\Gamma_{0}^{2}=\tau_{i}^{-1}+\tau_{*}^{-1},
\end{gathered}
$$

where

$$
\tau_{*}^{-1}=\tau_{z}^{-1}+\tau_{w}^{-1} .
$$

Thus, the pseudospin-triplet Cooperon modes are attenuated. Performing the integral over momentum, one thus obtains logarithmic weak-localization corrections at $B=0$,

$$
\delta \sigma=\frac{e^{2}}{\pi h} \theta\left(\frac{\tau_{\phi}}{\tau_{\mathrm{tr}}}, \frac{\tau_{\phi}}{\tau_{i}}, \frac{\tau_{\phi}}{\tau_{*}}\right),
$$


where

$$
\begin{aligned}
\theta & \left(\frac{\tau_{\phi}}{\tau_{\mathrm{tr}}}, \frac{\tau_{\phi}}{\tau_{i}}, \frac{\tau_{\phi}}{\tau_{*}}\right) \\
& =\left[2 \ln \frac{\tau_{\phi}}{\tau_{\mathrm{tr}}}-\ln \left(1+2 \frac{\tau_{\phi}}{\tau_{i}}\right)-2 \ln \left(1+\frac{\tau_{\phi}}{\tau_{i}}+\frac{\tau_{\phi}}{\tau_{*}}\right)\right],
\end{aligned}
$$

and $\tau_{\mathrm{tr}} \approx 2 \tau$. The dependence of the various scattering rates [Eqs. (14)-(16)] on the impurity-potential amplitudes $V_{i j}$ is given by Eq. (12). One can see that both the sign and the amplitude of the weak-localization corrections depend strongly on the impurity type.

As we will study in detail below, impurities with large correlation lengths mix valleys weakly, $1 / \tau_{i} \approx 0$, and therefore only attenuate two of the pseudospin triplet modes, in a similar way as the relaxation rate due to the warping term of Eq. (16). This effect leads to the vanishing of the weak-localization effect and a flat magnetoconductance.

Upon applying an external magnetic field, the weaklocalization corrections are suppressed. Solving the Cooperon equation in the presence of a magnetic field by summing over the Landau levels, one finds that $\Delta \sigma(B)=\sigma(B)-\sigma(B=0)$ is given by

$$
\Delta \sigma(B)=\frac{e^{2}}{\pi h} \underbrace{\overbrace{\left[F\left(\frac{\tau_{B}^{-1}}{\tau_{\phi}^{-1}}\right)\right.}^{\text {Pseudospin singlet }} \overbrace{\left.-F\left(\frac{\tau_{B}^{-1}}{\tau_{\phi}^{-1}+2 \tau_{i}^{-1}}\right)-2 \cdot F\left(\frac{\tau_{B}^{-1}}{\tau_{\phi}^{-1}+\tau_{i}^{-1}+\tau_{*}^{-1}}\right)\right]}^{\text {Pseudospin triplet }}}_{\text {Isospin singlet }},
$$

where the function $F(z)$ in Eq. (25) is given by

$$
F_{\text {full }}(z)=-\psi\left(\frac{1}{2}+\frac{\tau_{B}}{\tau_{\text {tr }}}\right)+\psi\left(\frac{1}{2}+\frac{1}{z}\right)+\ln \left[z \tau_{B} / \tau_{\text {tr }}\right],
$$

where $\psi(z)$ is the digamma function and the magnetic rate is

$$
\tau_{B}^{-1}=\frac{4 e D_{e} B}{\hbar} .
$$

For weak magnetic fields, one can use the simplified form [4]

$$
F(z)=\ln (z)+\psi\left(1 / 2+z^{-1}\right) .
$$

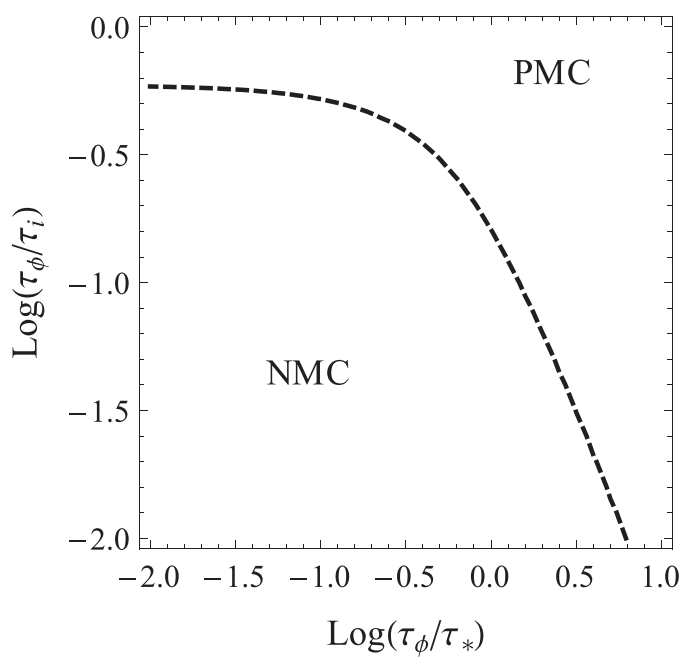

FIG. 1. $\tau_{\phi} / \tau_{*}-\tau_{\phi} / \tau_{i}$ diagram illustrating the transition from positive magnetoconductance (PMC) to negative magnetoconductance (NMC) as obtained from Eq. (30). The transition is indicated by the dashed black line, corresponding to $\Delta \sigma(B)=0$. This behavior is in good agreement with experiments [3].
Equation (25) can be expanded for small magnetic fields, yielding

$$
\Delta \sigma(B) \approx \frac{e^{2}}{24 \pi h}\left(\frac{4 e D B \tau_{\phi}}{\hbar}\right)^{2} \beta\left(\frac{\tau_{\phi}}{\tau_{*}}, \frac{\tau_{\phi}}{\tau_{i}}\right),
$$

where

$$
\beta\left(\frac{\tau_{\phi}}{\tau_{*}}, \frac{\tau_{\phi}}{\tau_{i}}\right)=\left[1-\frac{1}{\left(1+2 \frac{\tau_{\phi}}{\tau_{i}}\right)^{2}}-\frac{2}{\left(1+\frac{\tau_{\phi}}{\tau_{i}}+\frac{\tau_{\phi}}{\tau_{*}}\right)^{2}}\right] .
$$

In Fig. 1, we plot the curve obtained from the solution of $\Delta \sigma(B)=0$ as a function of the parameters $\tau_{\phi} / \tau_{*}$ and $\tau_{\phi} / \tau_{i}$. This curve separates the region of positive and negative magnetoconductance. We note that this separation line does not coincide with the curve $\delta \sigma(B=0)=0$, which corresponds to the vanishing of the weak-localization correction and separates the parameter space regions of weak localization and weak antilocalization (corresponding to negative and positive quantum corrections to the conductivity at $B=0$, respectively). As seen in Eq. (23), the latter separation line depends on an additional parameter, i.e., the total scattering rate $1 / \tau$.

\section{B. Thermopower}

Applying a thermal gradient $\nabla T$ to a metallic sample induces not only a thermal current but also an electrical current density, $\vec{j}=\sigma(\vec{E}+\nabla \mu / e)-\eta \nabla T$, where $\vec{E}$ is an applied electric field and $\mu$ is the chemical potential. Here, $\eta$ denotes the thermoelectric coefficient. Under open circuit conditions, the thermal gradient results in a finite voltage $U$ proportional to the temperature difference $\Delta T$, where the proportionality constant is the thermopower: $S=\eta / \sigma$. At low temperatures, the thermopower is dominated by the diffusion of the electrons in the sample and the phonon drag contribution becomes small. Expanding in the ratio of temperature to Fermi energy and keeping the leading term, one arrives at the Mott formula, which relates the thermopower $S$ to the derivative of the 
conductivity with respect to the Fermi energy,

$$
S=\frac{\pi^{2}}{3} \frac{k_{B}^{2} T}{e}\left[\frac{d \ln [\sigma(E)]}{d E}\right]_{E=E_{F}} .
$$

This formula is valid for low temperatures, $T \ll E_{F}$, and large chemical potential. For $T=0 \mathrm{~K}$, which is the case in this paper, the chemical potential is equal to the Fermi energy.

Here, $e=-|e|$ is the negative electron charge. Thus, when the carriers have negative charge, thermopower is expected to be negative, while for holes, it becomes positive. We note that the elemental unit of thermopower is given by a ratio of natural constants, $S_{0}=k_{B} /|e| \approx 86 \mu \mathrm{V} / \mathrm{K}$.

Furthermore, this formula is valid not only classically but also includes quantum corrections through the conductivity. Thus, expanding in the quantum correction to the conductivity $\delta \sigma$, we can write the leading quantum corrections to the thermopower, $\delta S$, as

$$
\frac{\delta S}{S}=-\frac{\delta \sigma}{\sigma}+\left[\frac{d \delta \sigma(E)}{d E}\right]_{E=E_{F}} /\left[\frac{d \sigma(E)}{d E}\right]_{E=E_{F}} .
$$

This relation is also supported by direct diagrammatic calculations of quantum corrections [10-12]. In standard metals, the last term on the right-hand side (r.h.s.) of Eq. (32) is small and the thermopower is dominated by the quantum correction to the conductivity. In this work, we revisit this relation to find out whether this also holds for graphene or whether the second term in Eq. (32) is sizable.

\section{THE RECURSIVE GREEN'S-FUNCTION METHOD}

In this section, we introduce the numerical method used to calculate the electrical conductivity and thermopower. We employ the recursive Green's-function method [20,21], which has been previously applied to graphene in Refs. [22-25]. Below, we begin by reviewing the essential elements of this method.

The graphene sample is assumed to be connected to two semi-infinite leads, which are modeled by a square lattice. When contacting the leads at the zigzag edges of the graphene sample, there is no wave-function mismatch between the propagating modes in the square lattice leads and the graphene sample, provided that a proper energy shift is used [26]. The graphene sample is sliced transversely into $N$ equal cells, with each cell containing $M$ sites. We study the transport as a function of $N$ by changing the length $L$ of the sample, and as a function of $M$ by changing the sample width $W$. When the free edges are of the armchair type, the sample dimensions are related to $N$ and $M$ by $\frac{L}{a_{0}}=\frac{\sqrt{3}}{2}\left(\frac{N}{2}-1\right)+\frac{\sqrt{3}}{6}$ and $\frac{W}{a_{0}}=M-1$, where $a_{0}=2.46 \AA$ is the lattice constant set as the distance between two atoms of the same sublattice. See Fig. 2.

\section{A. The conductivity}

In two dimensions, the electrical conductivity $\sigma$ is related to the conductance $G$ by the standard expression $\sigma=L G / W$ and thus can be expressed in terms of retarded Green's function

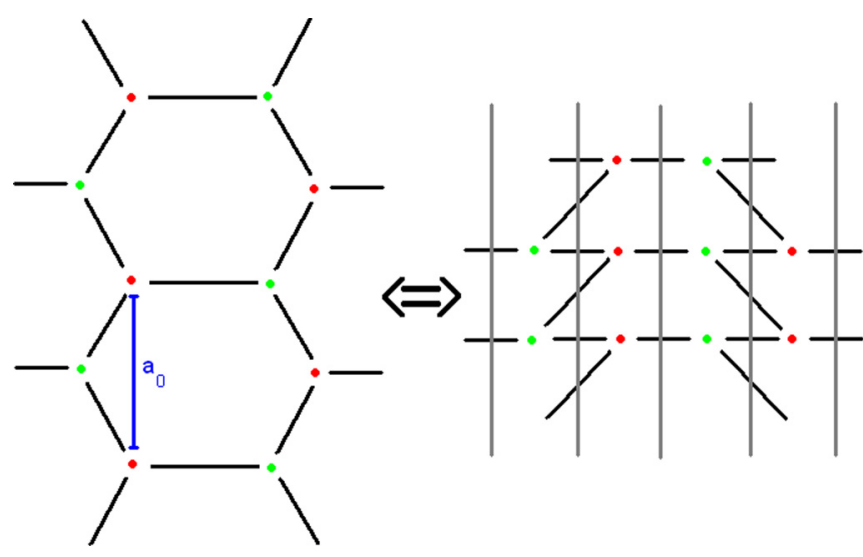

FIG. 2. (Color online) Slicing of graphene shown for two honeycomb cells when the contacts are at zigzag edges. Red and green dots indicate the two sublattices A and B; the connecting bonds of the sites are solid lines. The vertical slices, on the right side, are marked with vertical gray lines. $a_{0}$ represents the lattice constant, indicated in blue.

through the Caroli formula,

$$
\sigma=\frac{L}{W} \frac{2 e^{2}}{h} \operatorname{Tr}_{s}\left(\Gamma_{L} G^{R} \Gamma_{R} G^{A}\right),
$$

where $G^{A / R}$ denotes the advanced/retarded Green's function connecting two opposite contact regions (right and left). Here, $\operatorname{Tr}_{s}$ denotes the trace over transverse sites at the lead-sample interface. The matrix elements of the level width matrix $\Gamma_{p}$ can be expressed in term of the transverse wave functions $\chi_{v}(i)$ of the lead propagating modes,

$$
\Gamma_{p}\left(i, i^{\prime}\right)=\sum_{v} \chi_{v}(i) \frac{\hbar v_{v}}{a_{0}} \chi_{v}\left(i^{\prime}\right) .
$$

Here, the sum runs over the propagation modes $v$ and $v_{v}$ is the longitudinal propagation velocity. Next we describe the method used to obtain the Green's functions of the sample.

\section{B. The Green's function}

In order to obtain the Green's-function amplitude $G_{p q}^{R}(i, j)$ between sites $i, j$ at the contacts $p, q$, we start with the surface Green's function of one of the contacts, which is presumed to be known. Then we add one slice of transverse sites $i=$ $1, \ldots, M$ and calculate the Green's function from the contact up to that slice. We repeat the procedure, adding slice by slice, until the end of the sample is reached. This procedure is done from left to right and then in the opposite direction in order to calculate the full Green's function of the system. The slicing is displayed in Fig. 2.

Each line in Fig. 2 represents a hopping amplitude from one sublattice to another. This is described by the hopping matrix $U$. This matrix, together with the Green's function of every new single slice $g_{n}$ and the Green's function that includes the contacts and the sample up to slice $n$, defining $G^{(0)}$, are inserted in the Dyson equation $G=G^{(0)}+G^{(0)} V G$ to obtain the new $G$. One can easily understand that the speed of the procedure strongly depends on the slicing scheme adopted. In general, more slices mean more steps to calculate, but each 
slice consists of fewer atom sites and so its calculation is done faster since an inversion at each slice is required. The complexity of the calculation scales as $O\left(N M^{3}\right)$.

The Green's function of the contacts has been derived previously and is given by [27]

$$
G^{\mathrm{semi}}(v)=\frac{2 p_{v}}{\left(2\left|t^{x}\right|\right)^{2}}\left[1-\sqrt{1-\left(\frac{2\left|t^{x}\right|}{p_{v}}\right)^{2}}\right],
$$

with

$$
p_{v}=-V_{\text {gate }}+2\left|t^{y}\right| \cos \left(\frac{\pi v}{M+1}\right)+i 0^{+},
$$

where $v=1, \ldots, M$ represents the modes of the transverse wave function and $t^{i}$ is the hopping rate in the $i$ direction. We can transform $G^{\text {semi }}(v)$ from the channel representation into $G^{\text {semi }}\left(j, j^{\prime}\right)$ in the site representation by

$$
G^{\mathrm{semi}}\left(j, j^{\prime}\right)=\sum_{\nu=1}^{M} \chi_{\nu}^{*}(j) G^{\mathrm{semi}}(v) \chi_{\nu}\left(j^{\prime}\right),
$$

with the transverse wave functions $\chi_{v}(j)$ given by

$$
\chi_{v}(j)=\sqrt{\frac{2}{M+1}} \sin \left(\frac{\pi v j}{M+1}\right) .
$$

\section{Inclusion of impurities}

We now look at the model disorder used in the numerical calculations. The sites of the graphene sample are occupied by uniformly randomly distributed Gaussian scatterers with a random potential $V_{n} \in[-\delta V, \delta V]$. We can write, for the overall potential resulting from all $N_{\mathrm{imp}}$ scatterers at the points $\vec{R}_{n}$

$$
V\left(\vec{r}_{j}\right)=\sum_{n=1}^{N_{\text {imp }}} V_{n}\left(\vec{r}_{j}\right)=\sum_{n=1}^{N_{\text {imp }}} V_{n} e^{-\frac{\left|\overrightarrow{r_{j}}-\vec{R}_{n}\right|^{2}}{2 \xi^{2}}} .
$$

Here, $\xi$ is the range of the potential and $\vec{r}_{j}$ are the lattice sites. The concentration of scatterers is given by $n_{\text {imp }}=N_{\text {imp }} / N_{\text {tot }}$, where $N_{\text {tot }}$ is the total number of lattice sites. We focus on pairwise uncorrelated impurities, $\left\langle V_{n} V_{n^{\prime}}\right\rangle=\left\langle V_{n}^{2}\right\rangle \delta_{n, n^{\prime}}$, each vanishing on average, $\left\langle V_{n}\right\rangle=0$.

We use as an input parameter the dimensionless correlation strength $K_{0}$, which is defined by the equation for the impuritypotential correlation function as

$$
\left\langle V\left(\vec{r}_{i}\right) V\left(\vec{r}_{j}\right)\right\rangle=\frac{K_{0}\left(\hbar v_{F}\right)^{2}}{2 \pi \xi^{2}} e^{\frac{-\left|\vec{r}_{i}-\vec{r}_{j}\right|^{2}}{2 \xi^{2}}} .
$$

In the limit of dilute impurities, summing over all sites $i, j$ yields

$$
K_{0}=\frac{L W}{\left(\hbar v_{0}\right)^{2} N_{\text {tot }}^{2}} \sum_{i=1}^{N_{\text {tot }}} \sum_{j=1}^{N_{\text {tot }}}\left\langle V\left(\vec{r}_{i}\right) V\left(\vec{r}_{j}\right)\right\rangle .
$$

Inserting Eq. (39) into Eq. (41) and using the relations

$$
N_{\mathrm{tot}}=\frac{4 \sqrt{3}}{3} \frac{L W}{a_{0}} \text { and } v_{F}=\frac{\sqrt{3}}{2} \frac{t a_{0}^{2}}{\hbar},
$$

we get [28]

$$
K_{0}=(\delta V)^{2} \varrho
$$

where $\delta V$ is the half width of the box distribution of the amplitude of the Gaussian impurities $V_{n}$, defined in Eq. (39), and

$$
\varrho=\frac{\sqrt{3}}{9}\left(\frac{1}{t}\right)^{2} \frac{1}{N_{\mathrm{tot}}} \sum_{n=1}^{N_{\mathrm{imp}}}\left[\sum_{i=1}^{N_{\mathrm{tot}}} e^{\left(-\frac{\left(\overrightarrow{\vec{r}_{i}}-\overrightarrow{R_{n}}\right)^{2}}{2 \xi^{2}}\right)}\right]^{2} .
$$

Thus, the parameter $\varrho$ depends on $L, W$, and $\xi$ and is proportional to the concentration of impurities $n_{\text {imp }}=N_{\text {imp }} /(L W)$. It is useful to introduce the typical impurity strength amplitude $V_{\text {ta }}$, which is related to $K_{0}$ and $\varrho$ by

$$
V_{\mathrm{ta}} \equiv \sqrt{\left\langle V_{n}^{2}\right\rangle}=\sqrt{\frac{1}{3}(\delta V)^{2}}=\sqrt{\frac{1}{3} \frac{K_{0}}{\varrho}} .
$$

\section{CONNECTION BETWEEN THE TWO IMPURITY DESCRIPTIONS FROM SECS. III AND V C}

\section{A. Impurity potential}

In this section, we establish the link between the description of the impurities in graphene in the pseudospin and isospin representation, as introduced in Sec. III, and the Gaussian impurities introduced in the previous section [13,28]. McCann and co-workers assume that the different components of the impurity potential given by Eq. (11) are uncorrelated,

$$
\left\langle V_{i, j}(\vec{r}) V_{i^{\prime}, j^{\prime}}\left(\vec{r}^{\prime}\right)\right\rangle=V_{i, j}^{2} \delta\left(\vec{r}-\vec{r}^{\prime}\right) \delta_{i, i} \delta_{j, j},
$$

where $i, j$ denote the pseudospin and isospin indices as introduced in Eq. (11). We can decompose the potential due to one impurity at a given site of the lattice, $V_{n}(\vec{r})$, in Fourier components. Defining the vector connecting sublattices as $\vec{m}$, we find, for the Fourier component of $V_{n}(\vec{r})$ on sublattice A [13,29],

$$
V_{\vec{q}, n}=\frac{\sqrt{3} a_{0}^{2}}{2} \sum_{\vec{r}} V_{n}(\vec{r}) e^{-i / \hbar \vec{q} \vec{r}},
$$

and for sublattice $B$,

$$
V_{\vec{q}, n}^{\prime}=\frac{\sqrt{3} a_{0}^{2}}{2} \sum_{\vec{r}} V_{n}(\vec{r}-\vec{m}) e^{-i / \hbar \vec{q} \vec{r}},
$$

where the sum is over all elementary cells. We assume $V_{\vec{q}, n}$ and $V_{\vec{q}, n}^{\prime}$ to be slow functions of the momentum $\vec{q}$. Thus, the quantity $V_{\vec{q}, n}$ is proportional to the first-order scattering amplitude for electrons on the same sublattice where the impurity resides, while $V_{\vec{q}, n}^{\prime}$ is the one for electrons on the other sublattice. We will explicitly consider two values: the intravalley scattering, $q=0$, and the intervalley scattering, $q=k_{0}$, where $k_{0}$ connects the two different valleys in the reciprocal space and has the amplitude $k_{0}=2 h / 3 a_{0}$. We thus have $V_{0, n}$ and $V_{0, n}^{\prime}$ for intravalley scattering in $\mathrm{K}-\mathrm{K}^{\prime}$ space and $V_{k_{0}, n}$ and $V_{k_{0}, n}^{\prime}$ for intervalley scattering. Intravalley scattering means that the electron that is scattered does not leave the original cone-shaped valley in $k$ space, while in the intervalley scattering process, the electron starts in the $\mathrm{K}$ valley and ends after scattering in the $\mathrm{K}^{\prime}$ valley, or vice versa.

Equations (47) and (48) can now be displayed in the form of Eq. (11) using a $4 \times 4$ matrix notation. For impurities $V_{n}(\vec{r})$ located on sublattice A in the elementary cell $\overrightarrow{r_{n}}$ and small $\vec{q}$, 
we can approximate

$$
V_{\vec{q}, n}^{\mathrm{A}}=\left(\begin{array}{cccc}
V_{0, n} & 0 & 0 & V_{\vec{k}_{0}, n} e^{-2 i \overrightarrow{k_{0} \vec{r}_{n}}} \\
0 & V_{0, n}^{\prime} & 0 & 0 \\
0 & 0 & V_{0, n}^{\prime} & 0 \\
V_{\vec{k}_{0}, n} e^{2 i \vec{k}_{0} \vec{r}_{n}} & 0 & 0 & V_{0, n}
\end{array}\right) e^{-i \vec{q} \vec{r}_{n}}
$$

and, equivalently, for impurities in sublattice B,

$$
V_{\vec{q}, n}^{\mathrm{B}}=\left(\begin{array}{cccc}
V_{0, n}^{\prime} & 0 & 0 & 0 \\
0 & V_{0, n} & V_{\vec{k}_{0}, n} e^{-2 i \vec{k}_{0} \vec{r}_{n}} & 0 \\
0 & V_{\overrightarrow{k_{0}}, n} e^{2 i \vec{k}_{0} \vec{r}_{n}} & V_{0} & 0 \\
0 & 0 & 0 & V_{0, n}^{\prime}
\end{array}\right) e^{-i \vec{q} \vec{r}_{n}},
$$

where we used the fact that $V_{\overrightarrow{k_{0}}, n}^{\prime}$ vanishes due to the symmetry of graphene (otherwise, we would not have any zeros in the secondary diagonal, but terms involving $\left.V_{\overrightarrow{k_{0}}, n}^{\prime} e^{-2 i \overrightarrow{k_{0}} \vec{r}_{n}}\right)$.

As the next step, we convert Eqs. (49) and (50) into one single impurity-potential matrix that can be compared with Eq. (11). This is done by calculating the autocorrelation function $\left\langle\left\langle V_{\vec{q}} \otimes V_{-\vec{q}}\right\rangle\right\rangle$, with $V_{\vec{q}}=1 / 2 \sum_{n=1}^{N_{\text {imp }}}\left(V_{\vec{q}, n}^{\mathrm{A}}+V_{\vec{q}, n}^{\mathrm{B}}\right)$, set by

$$
\left\langle V_{q} \otimes V_{-q}^{T}\right\rangle=\frac{n_{\mathrm{imp}}}{2}\left\langle V_{\vec{q}, n}^{\mathrm{A}} \otimes V_{-\vec{q}, n}^{\mathrm{A}}+V_{\vec{q}, n}^{\mathrm{B}} \otimes V_{-\vec{q}, n}^{\mathrm{B}}\right\rangle,
$$

where the averaging $\langle\langle\cdot\rangle\rangle$ is with respect to the positions of the impurities and the impurity strength $V_{n}$, whereas the averaging $\langle\cdot\rangle$ is only with respect to $V_{n}$. The normalization factors are already included in the prefactor of the r.h.s. of Eq. (51).

A comparison of Eq. (51) with the matrix notation of the impurity, as introduced in Eq. (11), leads, with the help of Eq. (45) and the use of the impurity position averaged parameter $\langle\varrho\rangle$ from Eq. (44), to the following set of equations:

$$
\begin{gathered}
V_{0,0}{ }^{2}=1 / 4\left(V_{0}+V_{0}^{\prime}\right)^{2}, \\
V_{3,3}{ }^{2}=1 / 4\left(V_{0}-V_{0}^{\prime}\right)^{2}, \\
V_{\perp \perp}{ }^{2}=1 / 8 V_{\overrightarrow{k_{0}}}^{2},
\end{gathered}
$$

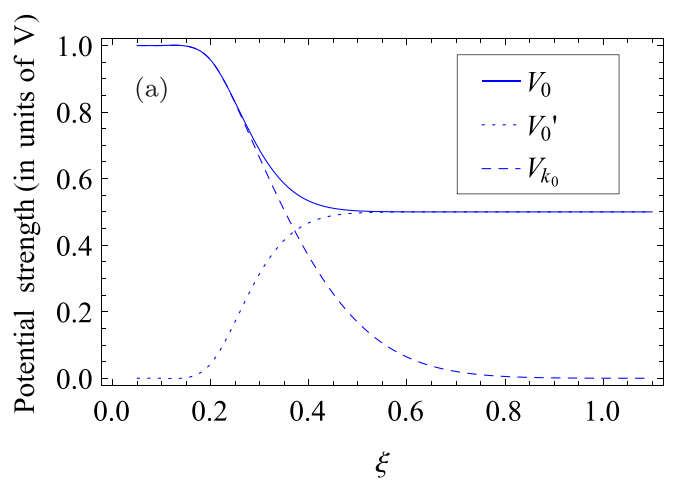

where

$$
\begin{gathered}
V_{0}=\sqrt{\left\langle V_{0, n}^{2}\right\rangle}=V / \tilde{\varrho} \sum_{\vec{r}} e^{-\frac{\vec{r}^{2}}{2 \xi^{2}}}, \\
V_{0}^{\prime}=\sqrt{\left\langle V_{0, n}^{\prime 2}\right\rangle}=V / \tilde{\varrho} \sum_{\vec{r}} e^{-\frac{(\vec{r}+\vec{m})^{2}}{2 \xi^{2}}}, \\
V_{\vec{k}_{0}}=\sqrt{\left\langle V_{\overrightarrow{k_{0}}, n}^{2}\right\rangle}=V / \tilde{\varrho} \sum_{\vec{r}} e^{-i \overrightarrow{k_{0} \vec{r}}} e^{-\frac{\vec{r}^{2}}{2 \xi^{2}}}, \\
V=\sqrt[4]{3} \sqrt{K_{0} t}, \\
\tilde{\varrho}=\sum_{\vec{r}}\left(e^{-\frac{\vec{r}^{2}}{2 \xi^{2}}}+e^{-\frac{(\vec{r}+\vec{m})^{2}}{2 \xi^{2}}}\right) .
\end{gathered}
$$

Note that $V$ is a measure of the total impurity strength averaged over all impurities. Since $K_{0}$ is proportional to their concentration $n_{\text {imp }}, V$ increases with $n_{\text {imp }}$ as

$$
V \sim \sqrt{n_{\mathrm{imp}}}
$$

In the limit of short-range impurities, $\xi \rightarrow 0$, we find

$$
\begin{aligned}
& V_{0}=V_{\overrightarrow{k_{0}}}=V, \\
& V_{0}^{\prime}=V_{\overrightarrow{k_{0}}}^{\prime}=0,
\end{aligned}
$$

while for long-range impurities, $\xi \rightarrow \infty$, we find

$$
\begin{gathered}
V_{0}=V_{0}^{\prime}=V / 2, \\
V_{\overrightarrow{k_{0}}}=V_{\overrightarrow{k_{0}}}^{\prime}=0 .
\end{gathered}
$$

In that limit, given by Eq. (63), only $V_{0,0}$ is not zero. We can see here that due to symmetry, $V_{\overrightarrow{k_{0}}}^{\prime}$ is always 0 and we are left with only one parameter $V$. This is shown in Fig. 3. Also displayed are the potential terms $V_{0}+V_{0}^{\prime}, V_{0}-V_{0}^{\prime}$, and $V_{\vec{k}_{0}}$ which, as we have seen above, are proportional to the impurity scattering matrix elements $V_{0,0}, V_{3,3}$, and $V_{\perp \perp}$.

Within the simplified picture of this section, the last result is again consistent with short-range scatterers mixing valleys and sublattices, while long-range scatterers mix only sublattices.

\section{B. Scattering rates}

We now calculate the scattering rates of Eqs. (14) and (15) and combine them with the potential Fourier components of Eqs. (52)-(54). (Note: We restrict ourselves here to the

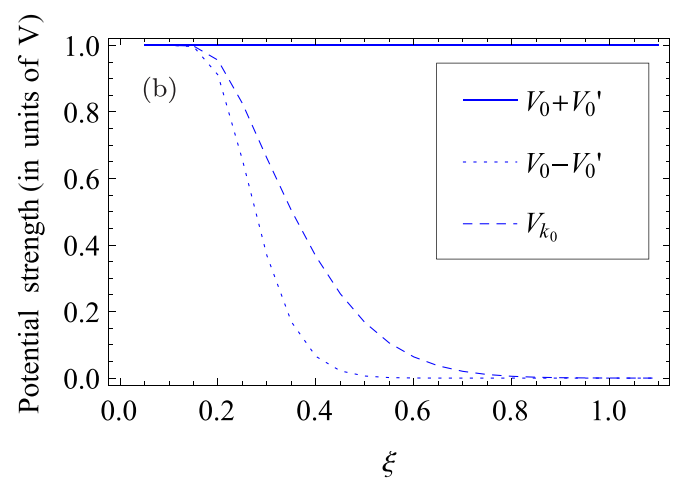

FIG. 3. (Color online) $V_{0}, V_{\overrightarrow{k_{0}}}$, and $V_{0}^{\prime}$ as set by Eqs. (49) and (50). (a) Effective potentials $V_{0}, V_{k_{0}}$, and $V_{0}^{\prime}$ in dependence on $\xi$. (b) Potential terms $V_{0}+V_{0}^{\prime}, V_{0}-V_{0}^{\prime}$, and $V_{k_{0}}$ in dependence on $\xi$. For short-range impurities, $V_{0}$ and $V_{\overrightarrow{k_{0}}}$ approach $V$ because the potential is localized only at the sites of one of the sublattices. Wider impurities also cover the neighboring sublattice and $V_{0}^{\prime}$ start to increase and $V_{0}, V_{k_{0}}$ decrease. Also visible is the faster decrease of $V_{0}-V_{0}^{\prime}$ than $V_{\overrightarrow{k_{0}}}$. $\xi$ in units of the lattice constant $a_{0}$. 
Born approximation. Going beyond that approximation, one needs to include all multiple impurity scattering, which yields corrections that depend logarithmically on energy [14] and can yield, according to Mott's law, additional contributions to thermopower.) The density of state per spin and per valley $v$, which is given by

$$
v=\frac{k_{F} A_{\mathrm{PC}}}{2 \pi \hbar^{2} v_{0}}=\frac{A_{\mathrm{PC}} E_{F}}{2 \pi \hbar^{2} v_{0}^{2}},
$$

is inserted into the general expression for the scattering rate, given by Eq. (12), where

$$
k_{F}=\frac{E_{F}}{v_{0}},
$$

and $A_{\mathrm{PC}}=a_{0}^{2} \sqrt{3} / 2$ is the area of the primitive unit cell.

In the case of Gaussian scatterers of Eqs. (52)-(54), and Eqs. (49) and (50), only the main- and off-diagonal matrix elements of Eq. (11) are nonzero. This helps us simplify Eqs. (14) and (15), so that we find

$$
1 / \tau_{3 \perp}^{-1}=1 / \tau_{\perp 3}^{-1}=0,
$$

resulting in

$$
\begin{aligned}
1 / \tau_{i} & =\frac{4}{\tau_{\perp \perp}}, \\
1 / \tau_{z} & =1 / \tau_{33} .
\end{aligned}
$$

Inserting Eqs. (52)-(54) and by using Eqs. (12) and (16) leads to

$$
\begin{gathered}
\frac{1}{\tau_{i}}=\frac{(1 / 2) \pi v V_{k_{0}}^{2}}{\hbar}, \\
\frac{1}{\tau_{z}}=\frac{(1 / 4) \pi v\left(V_{0}-V_{0}^{\prime}\right)^{2}}{\hbar}, \\
\frac{1}{\tau_{00}}=\frac{(1 / 4) \pi v\left(V_{0}+V_{0}^{\prime}\right)^{2}}{\hbar} .
\end{gathered}
$$

Now, using Eq. (64), we find

$$
\begin{gathered}
\frac{1}{\tau_{i}}=\frac{V_{k_{0}}^{2} A_{\mathrm{PC}} E_{F}}{4 \hbar^{3} v_{0}^{2}}, \\
\frac{1}{\tau_{z}}=\frac{\left(V_{0}-V_{0}^{\prime}\right)^{2} A_{\mathrm{PC}} E_{F}}{8 \hbar^{3} v_{0}^{2}}, \\
\frac{1}{\tau_{00}}=\frac{\left(V_{0}+V_{0}^{\prime}\right)^{2} A_{\mathrm{PC}} E_{F}}{8 \hbar^{3} v_{0}^{2}}, \\
\frac{1}{\tau_{w}}=\frac{16 \hbar^{2} \mu^{2} E_{F}^{3}}{\left(V_{0}+V_{0}^{\prime}\right)^{2} A_{\mathrm{PC}}} .
\end{gathered}
$$

Since the dephasing rate $1 / \tau_{\phi}=0$ in the numerical calculations, the low-energy cutoff is provided by the Thouless energy [30],

$$
E_{T}=\frac{D}{\Lambda^{2}}=\frac{v_{0}^{2} \tau_{T R}}{\Lambda^{2}}=\frac{4 \hbar^{3} v_{0}^{4}}{\left(V_{0}+V_{0}^{\prime}\right)^{2} \Lambda^{2} A_{\mathrm{PC}} E_{F}},
$$

where $\Lambda$ is the length or width of the sample, depending on which one is smaller, $\tau_{T R}=2 \tau_{0} \approx 2 \tau_{00}$, if we have $1 / \tau_{00} \gg$
$1 / \tau_{i j}$, for $i, j \neq 0$, and $D$ is the diffusion constant given by [31]

$$
D=v_{0}^{2} \tau_{T R} / 2
$$

Thus, we get the following ratios:

$$
\begin{gathered}
\frac{\tau_{\phi}}{\tau_{i}}=\frac{V_{k_{0}}^{2}\left(V_{0}+V_{0}^{\prime}\right)^{2} \Lambda^{2} A_{\mathrm{PC}}^{2} E_{F}^{2}}{32 \hbar^{6} v_{0}^{6}}, \\
\frac{\tau_{\phi}}{\tau_{z}}=\frac{\left(V_{0}-V_{0}^{\prime}\right)^{2}\left(V_{0}+V_{0}^{\prime}\right)^{2} \Lambda^{2} A_{\mathrm{PC}}^{2} E_{F}^{2}}{64 \hbar^{6} v_{0}^{6}}, \\
\frac{\tau_{\phi}}{\tau_{w}}=\frac{4 \mu^{2} \Lambda^{2} E_{F}^{4}}{\hbar v_{0}^{4}} .
\end{gathered}
$$

Since the factors $\left(V_{0}+V_{0}^{\prime}\right)^{2},\left(V_{0}-V_{0}^{\prime}\right)^{2}$, and $V_{\overrightarrow{k_{0}}}^{2}$ depend on the impurity parameters $V$ and $\xi$, as shown in Fig. 3, by tuning one of these parameters and the system size $\Lambda$, and Fermi energy $E_{F}$, one can move in the $\tau_{\phi} / \tau_{i}-\tau_{\phi} / \tau_{*}$ diagram of Fig. 1. This phase diagram is equivalent to what has been shown in the experimental paper [3], where the temperature has been varied to reach the different regions of the diagram. By tuning these parameters, a change from weak localization to weak antilocalization can be observed. We note that a change of the impurity concentration changes $\tau_{\phi} / \tau_{i}$ and $\tau_{\phi} / \tau_{z}$, while the ratio originating from the warping rate $\tau_{\phi} / \tau_{w}$ is independent of the impurity concentration $n_{\text {imp. }}$.

For the magnetic rate $1 / \tau_{B}$, we find, accordingly,

$$
\frac{1}{\tau_{B}}=\frac{64 B e \hbar^{2} v_{0}^{4}}{\left(V_{0}+V_{0}^{\prime}\right)^{2} A_{\mathrm{PC}} E_{F}} .
$$

\section{NUMERICAL RESULTS}

All results presented in the following are for samples with armchair edges. Most calculations have also been done with zigzag edges, especially in the case of small systems, but they do not show any significant difference and are, consequently, not displayed here. We consider always samples with an aspect ratio of $1, L \approx W$, ranging from $N=20$ and $M=48$; $M=30, N=72$; up to $M=80, N=192$. The correlation length $\xi$ is given in units of $a_{0}$, the lattice constant. The correlated disorder strength $K_{0}$ is the dimensionless parameter, defined in Eq. (43). For small systems, we use $\xi / a_{0}=$ $0.5, \ldots, 3$ and $K_{0}=0.5, \ldots, 4$, while for the larger system, we consider only $\xi / a_{0}=0.5, \ldots, 2$ and $K_{0}=0.5, \ldots, 2$. Unless explicitly mentioned otherwise, the impurity density is set to $n_{\text {imp }}=0.03$, meaning that $3 \%$ of the atomic sites are occupied by impurities. The experiments on weak antilocalization [3] indicate, indeed, that this is a realistic concentration of impurities in these graphene samples. We also performed the calculations for higher densities up to $n_{\mathrm{imp}}=0.3$.

Impurities are uniformly distributed across the sample. For each impurity realization, we calculate the electrical conductivity as delineated above. We run the numerical calculations for $N_{c}=5000$ different realizations in order to average the results for the conductivity and thermopower. For the larger systems, $N_{c}=1000$ turns out to be sufficient, since self-averaging improves with increasing system size. The Fermi energy $E_{F}$ is displayed in units of the hopping parameter $t$, which is set to $2.7 \mathrm{eV}$. We consider the range 


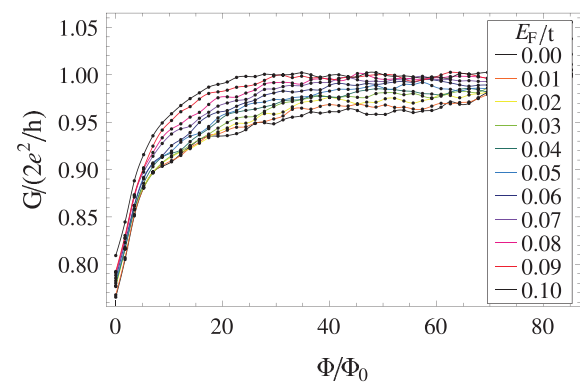

(a) $K_{0}=4, \xi=1$.

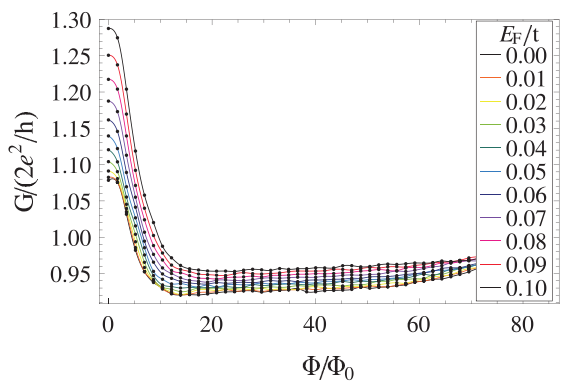

(b) $K_{0}=4, \xi=3$.

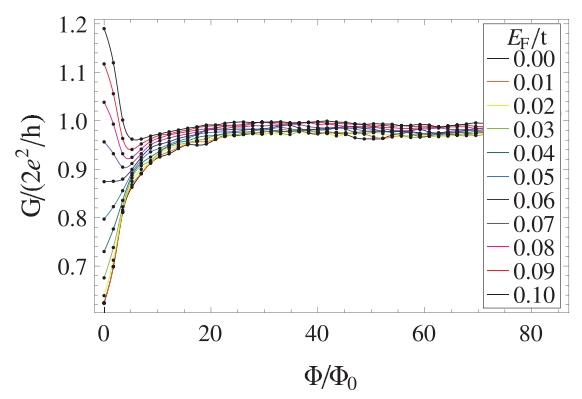

(c) $\xi=0.5, K_{0}=0.5, n_{\text {imp }}=0.3$.

FIG. 4. (Color online) Average conductance $G$, as calculated numerically with the recursive Green's-function method for dimensions $N=30$ and $M=72$. The Fermi energy $E_{F}$ is varied as indicated in the figure. (a) Positive magnetoconductance, weak-localization effect at small correlation length $\xi=1$. (b) Negative magnetoconductance, weak-antilocalization effect at larger correlation length $\xi=3$. (c) Change between positive and negative magnetoconductance by tuning $E_{F}$. The lines are a guide for the eye.

$E_{F} / t=0, \ldots, 0.1$, where $E_{F}=0$ corresponds to the Dirac point. The magnetic field is displayed as the magnetic flux $\Phi$ through the whole sample in units of the magnetic flux quantum $\Phi_{0}$.

\section{A. Conductivity}

\section{Magnetoconductance sign}

Numerical results for the magnetoconductivity are displayed in Fig. 4. We can distinguish the weak localization from the weak antilocalization by the sign of the magnetoconductance:

$$
\Delta \sigma_{\text {sgn }}(B)=\operatorname{sgn}\left(\frac{d \Delta \sigma(B)}{d B}\right) .
$$

Negative magnetoconductance $\Delta \sigma_{\mathrm{sgn}}(B)=-1$ at weak magnetic fields $B$ corresponds to weak antilocalization, since the magnetic field reduces the quantum correction and thereby the conductance. Positive magnetoconductance $\Delta \sigma_{\text {sgn }}(B)=1$ corresponds to weak localization. As seen in Figs. 5, this sign can change at large magnetic fields. This occurs as soon as the magnetic rate $1 / \tau_{B}$ exceeds all symmetry-breaking rates $1 / \tau_{i j}$ defined in Eq. (12). We note that the two-dimensional samples considered in the numerical calculations are rather small, with length $L$ and width $W$ that are much smaller than the magnetic length $l_{B}$ for moderate magnetic fields $B\left(l_{B}=0.026 \mu \mathrm{m} \sqrt{T / B}\right)$. Therefore, the sensitivity of the conductivity to an external magnetic field $B$ is reduced and the magnetic rate $1 / \tau_{B}$ becomes suppressed for $l_{B} \gg L, W$ to [32]

$$
1 / \tau_{B}=c D \frac{L W}{l_{B}^{4}},
$$

where $c$ is a geometrical factor of order unity. On the other hand, the symmetry-breaking rates $1 / \tau_{i j}$, given by Eq. (12), do not depend on the system dimensions $L$ and $W$. Only the relaxation rate originating from the warping term, $1 / \tau_{w}$, acquires a small sample size dependence and becomes smaller when $D \tau_{w} \gg L W$. Therefore, as seen in Fig. 4, the change of the weak-localization corrections occurs only when the magnetic fluxes through the samples correspond to huge magnetic fields. This is due to the small samples considered in the numerical calculations.
As seen in Fig. 4(a), for small correlation length, one observes positive magnetoconductance, while at larger correlation lengths, negative magnetoconductance occurs for the same impurity strength $K_{0}$; see Fig. 4(b). As we will analyze in detail below, this can be explained by the reduction of the intervalley scattering amplitude $V_{k_{0}}$ with the increase of the correlation length $\xi$, as shown in Fig. 3 .

A transition between positive and negative magnetoconductance is observed as a function of the Fermi energy $E_{F}$, as seen in Fig. 4(c). Similar transitions can be observed when changing $K_{0}$ or $n_{\text {imp }}$, which we do not show here.

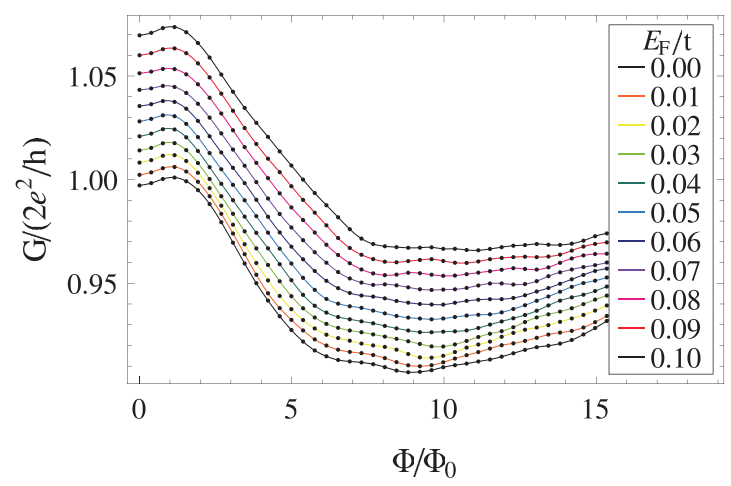

(a) Size $10 \times 24, \xi=1.5, \mathrm{~K}=4$

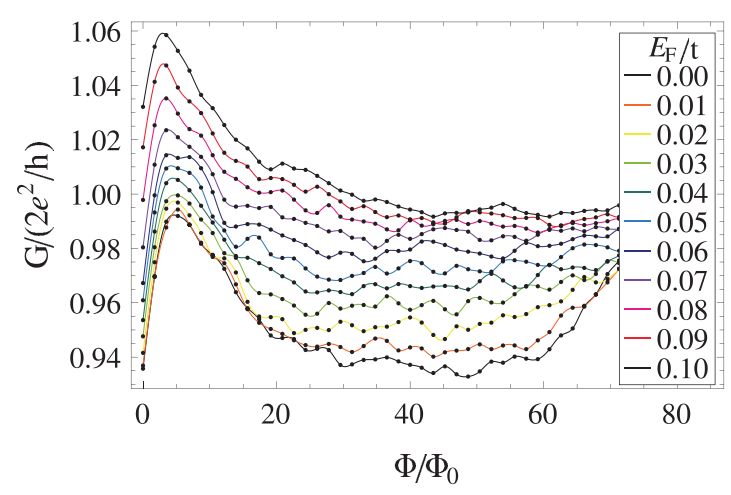

(b) Size $20 \times 48, \xi=1, \mathrm{~K}=2$

FIG. 5. (Color online) Samples of numerical results showing strongly nonmonotonic magnetoconductance. 


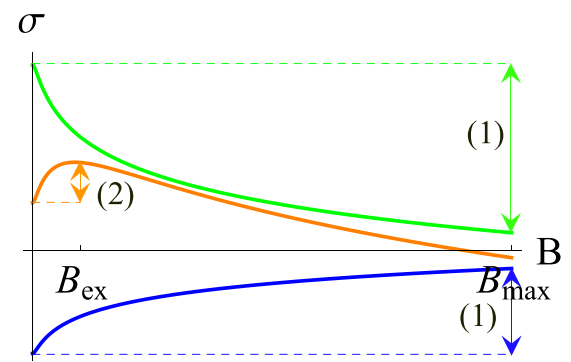

FIG. 6. (Color online) Schematic plots of different types of magnetoconductance $\sigma(B)$. At $B_{\max }$, all weak-localization corrections are suppressed. If the magnetoconductance is monotonous as in cases (1), the amplitude is $\Delta \sigma\left(B_{\max }\right)$. Otherwise, the magnetoconductance has a maximum at $B=B_{\mathrm{ex}}$, as in case (2).

\section{Magnetoconductance amplitude}

In addition to the sign of the magnetoconductance, the amplitude of the magnetoconductance $\Delta \sigma$ is important and can reveal more information on the nature of the impurities in the sample. As a measure of the amplitude of the magnetoconductance, we take the difference between the conductance at the first extremum $B_{\mathrm{ex}}$ and the one at zero magnetic field $(B=0)$,

$$
\Delta \sigma\left(B_{\mathrm{ex}}\right):=\sigma\left(B_{\mathrm{ex}}\right)-\sigma(B=0) .
$$

Positive $\Delta \sigma\left(B_{\mathrm{ex}}\right)$ thus means that there is a weak-localization dip at weak magnetic fields, while negative values correspond to the amplitude of the weak-antilocalization peak. We summarize the different types of magnetoconductivity schematically in Fig. 6. The amplitude $\Delta \sigma\left(B_{\mathrm{ex}}\right)$ is indicated by arrows, both in the case when there is no sign change $\Delta \sigma_{\text {sgn }}(B)$ as the magnetic field is increased (1), as well as when the sign changes (2). The quantum conductance corrections vanish at large magnetic fields, when $l_{B}$ is of the order of the elastic mean free path $l_{e}$. This magnetic field we denote as $B_{\max }$, as defined by $l_{B_{\max }}=l_{e}$.

As seen in Fig. 4, there are still statistical fluctuations on smaller magnetic field scales, despite the large number of realizations $N_{c}=5000$, which we used in the averaging of the conductance.

\section{Phase diagrams}

a. Magnetoconductance sign. In order to study the crossover between positive and negative magnetoconductance as a function of the three impurity parameters $\xi, K_{0}$, and $E_{F}$, we use Eq. (82) at weak magnetic fields to assign the value $\Delta \sigma_{\text {sgn }}(B \rightarrow 0)=-1$ for negative magnetoconductance $(\mathrm{NMC})$ and $\Delta \sigma_{\mathrm{sgn}}(B \rightarrow 0)=1$ for positive magnetoconductance (PMC). With this information, we create three such sign phase diagrams, varying two parameters while the third parameter stays fixed, see Fig. 7. For better visualization, the diagrams are colored yellow for negative and red for positive magnetoconductance. The crossing line is highlighted by the black line. We note that the numerical data is given only at the crossings of grid lines. We use an interpolation method to get the continuous crossing line. As seen in the $\xi-K_{0}$ diagram, Figs. 7(a) and 7(b), PMC occurs for short-range impurities with $\xi<a_{0}$ in the whole range of impurity strengths $K_{0}$. This is expected since short-range

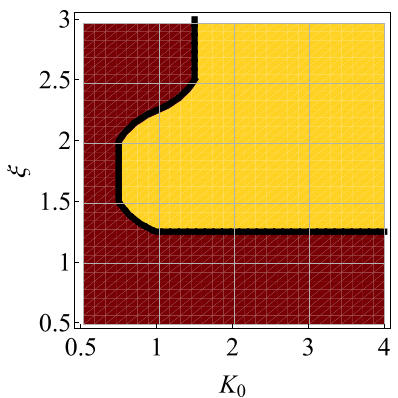

(a) $E_{F}=0.01 t$

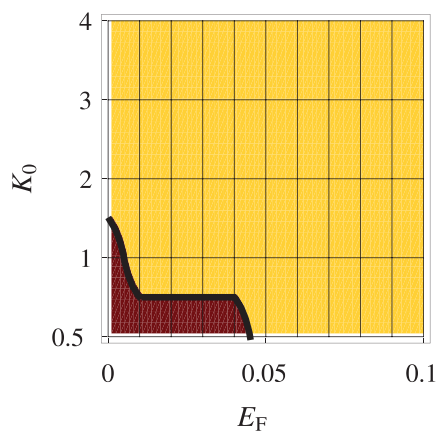

(c) $\xi=2 a_{0}$

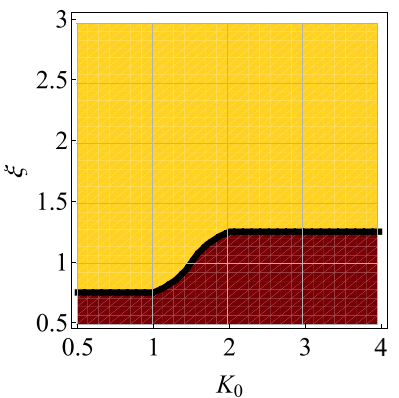

(b) $E_{F}=0.02 t$

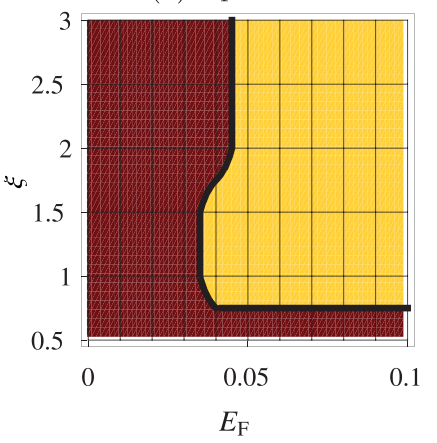

(d) $K_{0}=0.5$
FIG. 7. (Color online) Numerical magnetoconductance sign phase diagrams calculated with Eq. (82): (a),(b) as a function of correlation length $\xi$ and impurity strength parameter $K_{0}$ at fixed Fermi energy, (c) as a function of $K_{0}$ and $E_{F}$ for fixed $\xi$, and (d) as a function of $\xi$ and $E_{F}$ for fixed $K_{0}$. Positive magnetoconductance is indicated by the red area, while negative magnetoconductance is yellow. The system size is fixed to $M=30$ and $N=72$.

impurities cause intervalley scattering and thereby suppress the pseudospin-triplet Cooperons.

For impurities with larger correlation lengths $\left(\xi>a_{0}\right)$, NMC is observed for strong $K_{0}$ and large $E_{F}$, as seen in Fig. 7(c). Surprisingly, there is a regime at weak $K_{0}$ and small $E_{F}$ where PMC occurs even for large $\xi$, see Fig. 7(d). We observed similar behavior for other sizes $M$ and $N$, which we do not display here. In the $E_{F}-K_{0}$ diagram, we see a change from PMC to NMC when moving away from the Dirac point. At the Dirac point, we observe a change at $K_{0} \approx 1$. For $E_{F}>0.05 \mathrm{t}$, only NMC is observed for $\xi=2 a_{0}$. The $\xi-E_{F}$ phase diagram shows that for $\xi \ll a_{0}$, PMC occurs independent of the Fermi energy. At larger $\xi$, a change to NMC occurs, as expected.

In order to get a simpler representation of the results, we merge all three two-dimensional phase diagrams into one three-dimensional phase diagram, in which each of the parameters $\xi, K_{0}$, and $E_{F}$ is represented by one axis; see Fig. 8.

b. Magnetoconductance amplitude. Using Eq. (84), we determine the amplitude of the magnetoconductance and plot it in contour plots in the $\xi-K_{0}$, the $E_{F}-K_{0}$, and the $\xi-E_{F}$ plane, respectively, in Fig. 9. In these figures, the contour lines are tagged with the corresponding numbers calculated from Eq. (84). The main features already observed in the magnetoconductance sign phase diagrams can be seen in Fig. 9: PMC occurs for short-range impurities with $\xi \ll$ $a_{0}$, irrespective of $K_{0}$ and $E_{F}$. For impurities with larger 


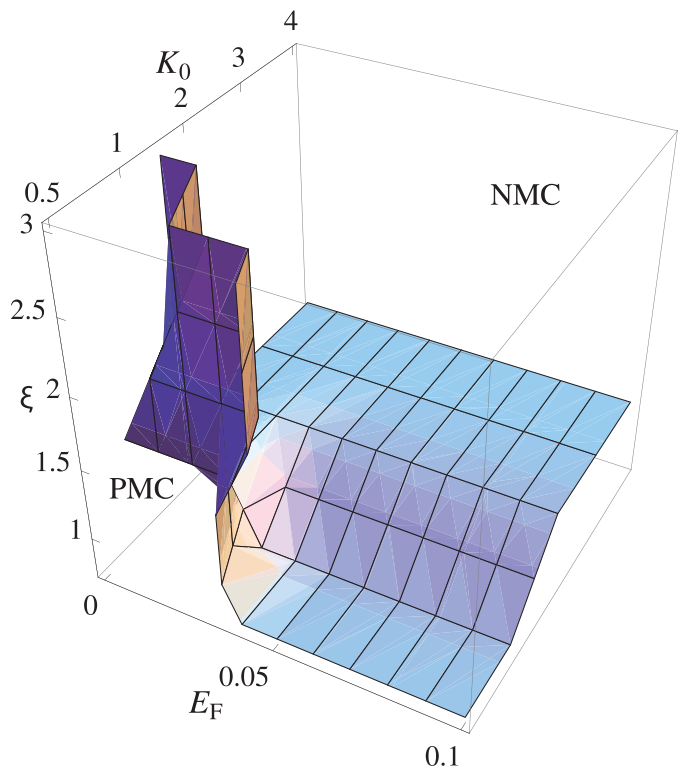

FIG. 8. (Color online) $E_{F}-K_{0}-\xi$ phase diagram for $M=30$ and $N=72$, where the magnetoconductance sign is obtained from the numerical results with Eq. (82). The surface with vanishing magnetoconductance is displayed.

correlation lengths $\left(\xi>a_{0}\right)$, there is NMC for strong $K_{0}$ and large $E_{F}$, as expected. At weak $K_{0}$ and small $E_{F}$, PMC occurs even for large $\xi$. Its amplitude remains weak, however, corresponding to only a small weak-localization dip at weak magnetic fields. This is displayed in Fig. 10.

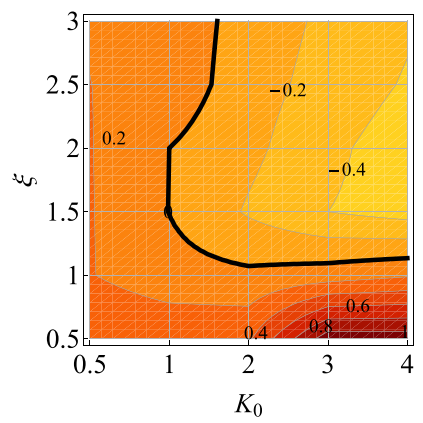

(a) $E_{F}=0.01 t$

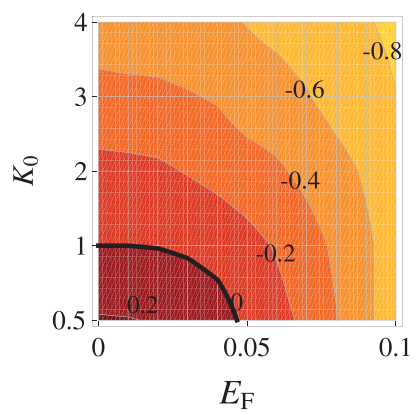

(c) $\xi=2$

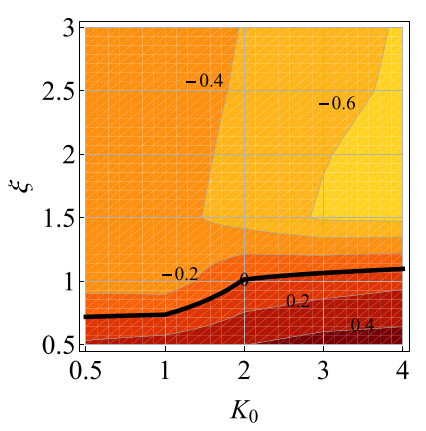

(b) $E_{F}=0.1 t$

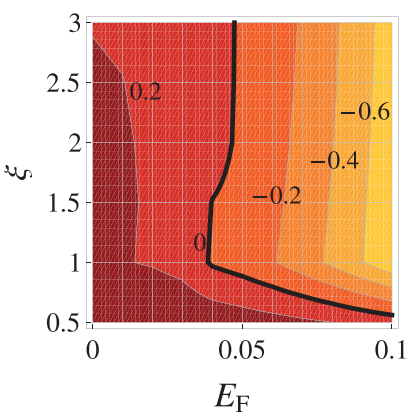

(d) $K_{0}=0.5$
FIG. 9. (Color online) Magnetoconductance amplitude obtained from the numerical results with Eq. (84) in (a),(b) the $\xi-K_{0}$ for two $E_{F}$, (c) the $K_{0}-E_{F}$, and (d) the $\xi-E_{F}$ plane, respectively. The black line indicates vanishing magnetoconductance. Contour lines are in units of $2 e^{2} / h . M=30$ and $N=72$.

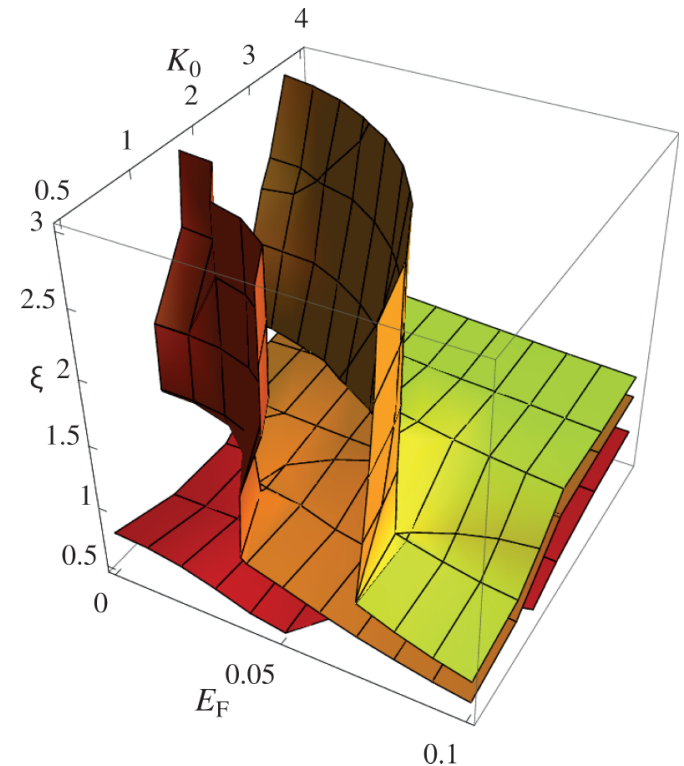

FIG. 10. (Color online) Numerical results as a function of the parameters $E_{F}, K_{0}, \xi$ for the magnetoconductance amplitude, given by Eq. (84), for $M=30$ and $N=72$. Surfaces of value 0.3 (red), 0 (orange), and -0.3 (yellow) are displayed, in units of $2 e^{2} / h$. Each grid line crossing corresponds to a numerically calculated value.

\section{B. Thermopower}

Next, we calculate the thermopower $S$, as introduced in Sec. IV B, using the Mott formula of Eq. (31) in order to see if there are quantum corrections and, as a result, magnetothermopower. To this end, we use the results for the conductance $G$ and its dependence on Fermi energy $E_{F}$ from the previous section to calculate and analyze $\frac{d G}{d E_{F}}$ and the thermopower $S$. We note that by expanding in the quantum corrections of the conductivity to first order, we can write the quantum correction to the thermopower $\delta S$ as

$$
\frac{\delta S}{T}=-\frac{S_{\mathrm{cl}}}{T} \frac{\delta G}{G_{\mathrm{cl}}}-\frac{\pi^{2}}{3} \frac{k_{B}^{2}}{|e|} \frac{\delta d G / d E}{G_{\mathrm{cl}}},
$$

where $\frac{\pi^{2}}{3} \frac{k_{B}^{2}}{|e| e V}=0.024 \mu \mathrm{V} / \mathrm{K}^{2}$. Since the conductance change with magnetic field is only of the order of $e^{2} / h$, the first term in Eq. (85) is of the order of $\frac{S_{\mathrm{cl}}}{T}$. Moreover, since $S_{\mathrm{cl}}$ vanishes at the Dirac point and increases linearly with the gate voltage, we expect the last term to become more important in the vicinity of the Dirac point. This is confirmed by the numerical results shown in Fig. 11(b), where $\frac{d G}{d E_{F}}$ is plotted as a function of magnetic field. This quantity decays with magnetic field; the larger the Fermi energy $E_{F}$, the larger is the sensitivity to the magnetic field. As shown in Fig. 11(a), the thermopower shows the same qualitative behavior, namely, a reduction of the amplitude of the thermopower when a magnetic field is applied. For positive gate voltage, the thermopower is negative, so that we obtain positive magnetothermopower.

For the largest samples considered, we also observe an initial increase of $\frac{d G}{d E_{F}}$ at weak magnetic fields, and a peak which becomes enhanced with increasing Fermi energy (see Fig. 12), followed by a decay to its classical value at larger magnetic fields. Correspondingly, in these samples, the 


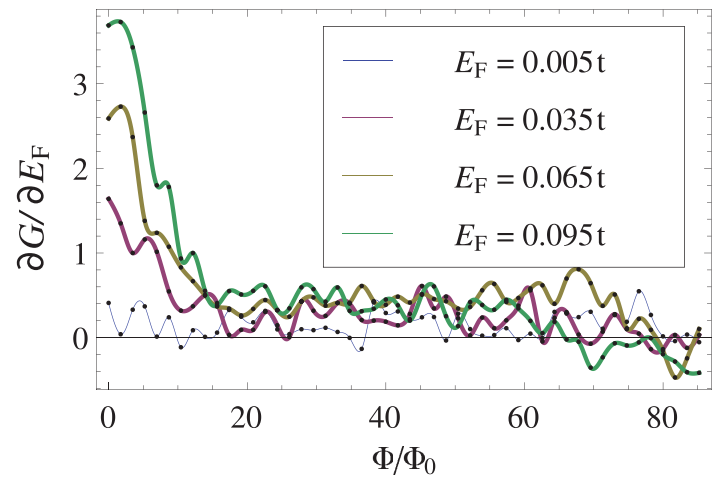

(a) $\xi=2$

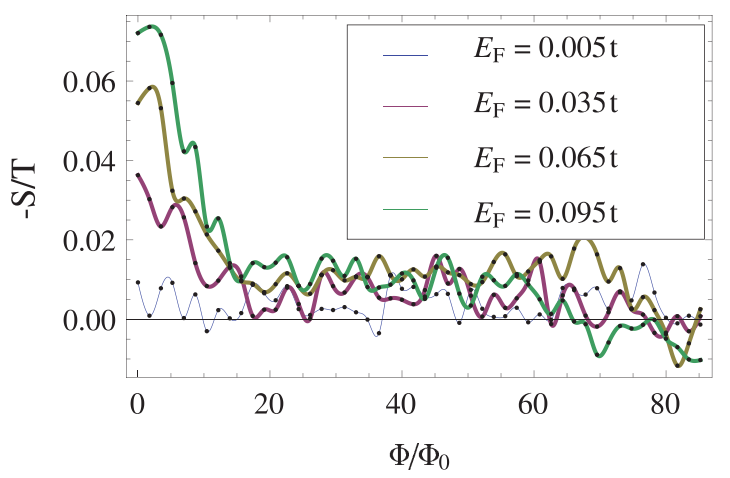

(b) $\xi=2$

FIG. 11. (Color online) Numerical results for (a) $d G / d E_{F}$ in units of $\left(2 e^{2} / h\right) / \mathrm{eV}$ as a function of magnetic flux $\phi(M=30$, $N=72, K_{0}=2$ ). (b) Magnetothermopower in units of $\mu \mathrm{V} / \mathrm{K}^{2}$ for the same system as (a). The corresponding conductivity is shown in Fig. 4(b).

amplitude of the thermopower [see Fig. 12(c)] first is enhanced when the magnetic field is applied and then decreases toward its classical value at larger magnetic fields.

Since the magnetic field suppresses quantum corrections, we can obtain the classical value numerically from the high magnetic field limit. Since the thermopower is fluctuating even in the range where it should saturate towards the classical limit, we needed to average over the fluctuations of the data points at different magnetic fields. For every set of parameters, we thereby calculate $S_{\text {mean }}$ and use as a definition

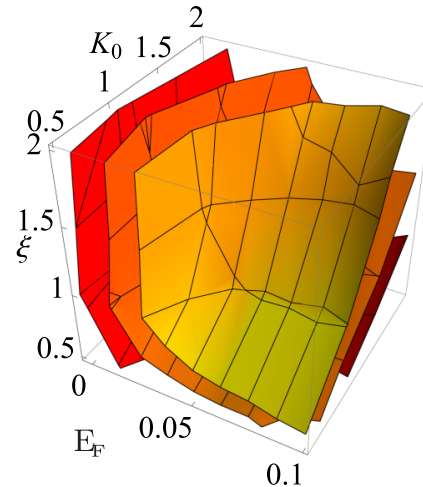

(a) $M=60$ and $N=144$.

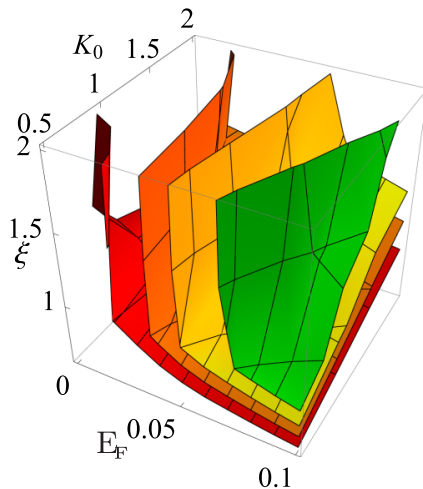

(b) $M=60$ and $N=144$.

FIG. 13. (Color online) Numerical results for (a) magnetothermopower amplitude $\Delta S / T$, given by Eq. (86), as a function of $E_{F}, K_{0}, \xi$. Surfaces with value -0.05 (red), -0.1 (orange), and -0.2 (yellow) in units of $\mu \mathrm{V} / \mathrm{K}^{2}$ are displayed. (b) Magnetoconductance amplitude $\Delta \sigma$, given by Eq. (84), as a function of the parameters $E_{F}, K_{0}, \xi$. Surfaces of value 0 (red), -0.3 (orange), -0.6 (yellow), and -1 (green) in units of $2 e^{2} / h$ are displayed. (The grid corresponds to the numerically calculated values.)

for the quantum corrections the difference to the thermopower without magnetic field,

$$
\Delta S:=S(B=0)-S_{\text {mean }} .
$$

In the following, we explore the size and sign of these quantum corrections of the thermopower and how they depend on the impurity parameters, such as the strength $K_{0}$ and the correlation length $\xi$, and on the Fermi energy. In Fig. 13(a), we plot the values of $\Delta S$ as a function of these three parameters. As we are particularly interested in how these quantum corrections to the thermopower are related to the weak-localization corrections to the conductivity, we also plot the magnetoconductance amplitude (MCA) in Fig. 13(b) in units of $2 e^{2} / h$. We notice that the set of parameters giving the most pronounced weak antilocalization seems also to yield the strongest thermopower enhancement. We will explain and analyze this effect in detail in Sec. VIIID. This behavior can also be seen when looking at the MC amplitude phase diagrams as a function of $E_{F}$ in Fig. 9, since the derivative $\frac{d G}{d E_{F}}$ is the highest when there is a strong increase of values in the $E_{F}$ direction. This is the case in the regime of weak

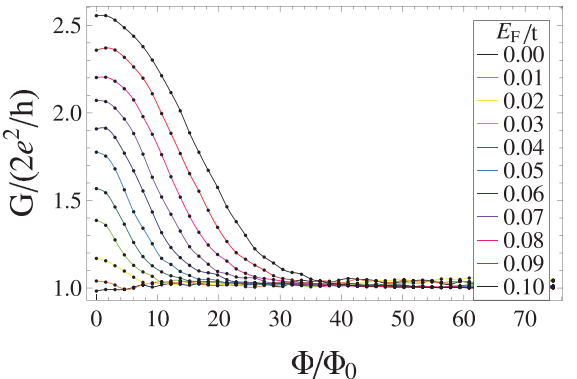

(a) $M=80, N=192$

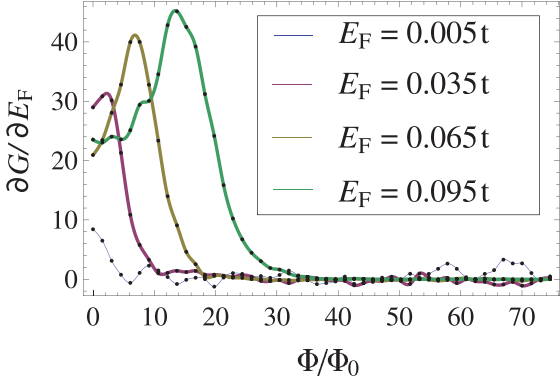

(b) $M=80, N=192$

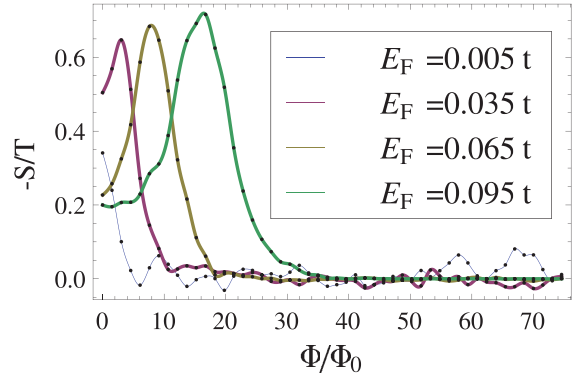

(c) $M=80, N=192$

FIG. 12. (Color online) Numerical results for (a) conductance, (b) $d G / d E_{F}$ in units of $\left(2 e^{2} / h\right) / \mathrm{eV}$, and (c) magnetothermopower in units of $\mu \mathrm{V} / \mathrm{K}^{2}\left[(\mathrm{a})-(\mathrm{c}): K_{0}=0.5, \xi=2\right]$. 
antilocalization, while a smaller change is observable in the weak-localization regime; see Figs. 9(c) and 9(d).

\section{ANALYSIS OF NUMERICAL RESULTS}

In order to get a better understanding of these numerical results for the magnetoconductance and the magnetothermopower, we compare them with the analytical expression of Eq. (25) and use the scattering rates $1 / \tau_{z}$ and $1 / \tau_{i}$ and the effective dephasing rate $1 / \tau_{\phi}$ as fitting parameters. We also attempt an $a b$ initio calculation where we use the input parameters of the numerical calculations to calculate directly the different scattering rates $1 / \tau_{i j}$, insert these in the analytical expression, and compare the result with the numerical ones.

\section{A. Fitting of the numerical results}

For the fitting procedure, we picked the sample size $N=30$ and $M=72$, where we observed a clear transition between weak antilocalization and weak localization when changing the parameters $K_{0}, \xi$ and the Fermi energy; see Fig. 9. As mentioned before, since the magnetic length exceeds the system size $L$ in the magnetic flux range considered, we have to use the appropriate magnetic scattering rate $1 / \tau_{B}$, given by Eq. (83), which is strongly reduced by a geometrical factor compared to the two-dimension limit. As discussed above, since the numerical results are done at zero temperature, the dephasing rate $1 / \tau_{\phi}$ can be substituted by the Thouless energy; see Eq. (76).

\section{Fitting with a fixed $1 / \tau_{\phi}$}

In this section, the two fitting parameters in Eq. (25) are taken to be $1 / \tau_{z}$ and $1 / \tau_{i}$, while $1 / \tau_{\phi}$ is calculated directly from Eq. (76). For the parameter range $\xi / a_{0}=1,1.5,2$, $K_{0}=1,1.5,2$, and $E_{F} / t=0.01,0.05$, we find that Eq. (25) fits well. Within this parameter region, we find the transition line between weak antilocalization and weak localization. We concentrate our fitting procedure to the validity range of the analytical formula at small magnetic flux, where $l_{B}>L$, given by Eq. (83).

We show the results of the fitting exemplary for some parameters in Fig. 14(a), where the magnetoconductance is positive. In that case of pronounced weak localization, the fitting becomes worse and the uncertainty in the set of $1 / \tau_{i}$ and $1 / \tau_{z}$ is larger.

For each parameter set used in the numerical calculations, we get a set of fitted values of the rates $\tau_{\phi} / \tau_{i}-\tau_{\phi} / \tau_{z}$ which we plot in the diagrams of Figs. 15(a) and 15(b) as a symbol. The color of the symbols indicates the value of $K_{0}$, while the symbol itself depends on the correlation length as shown in the legend. Weak localization corresponds to empty symbols and weak antilocalization corresponds to filled symbols. The range of scattering rates is $\log \left(\tau_{\phi} / \tau_{i}\right) \approx-4, \ldots, 0$ and $\log \left(\tau_{\phi} / \tau_{z}\right) \approx$ $1, \ldots, 4$. Thus, the intervalley scattering rates are typically several magnitudes smaller than the intravalley scattering rates for the range of $\xi$ considered. The black line is obtained from $\Delta \sigma(B)=0$ in Eq. (30), indicating the transition between weak antilocalization and weak localization. With the exception of a few data points, we find good agreement. We can improve the agreement by also fitting $\tau_{\phi}$, as shown in the next section.

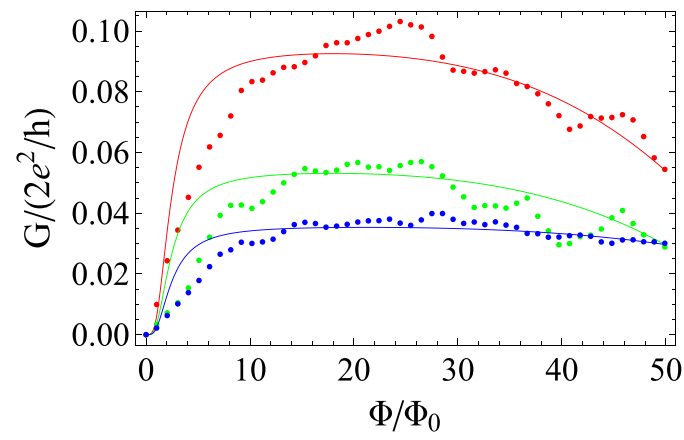

(a) $\tau_{\phi}$ calculated with Eq. (76)

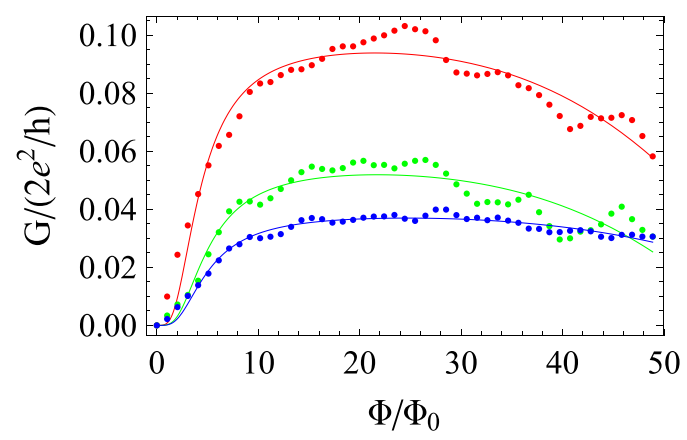

(b) $\tau_{\phi}$ fitted

FIG. 14. (Color online) The numerical results for the magnetoconductance amplitude $\Delta G(\phi)=G(\phi)-G(0)$ for $M=30, N=72$ together with the fitting in terms of $1 / \tau_{i}, 1 / \tau_{z}$ in Eq. (25). $\operatorname{Red} \xi=1$, green $\xi=1.5$, blue $\xi=2, E_{F}=0.01, K_{0}=1$.

\section{Fitting $1 / \tau_{\phi}$}

We repeat the fitting procedure for the same sample, but now take $1 / \tau_{\phi}$ as a third fitting parameter. We focus on the range of parameters $\xi / a_{0}=1,1.5,2, K=1,1.5,2$, and $E_{F} / t=0.01,0.05$. Close to the transition, the extra fitting
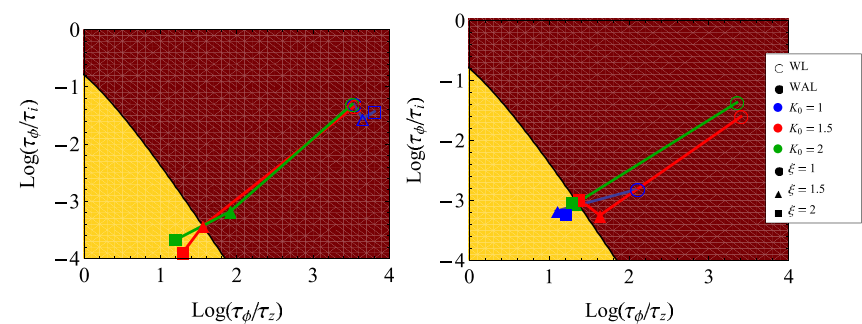

(a) $\tau_{\phi}$ from Eq. (76), $E_{F}=0.01 t$.
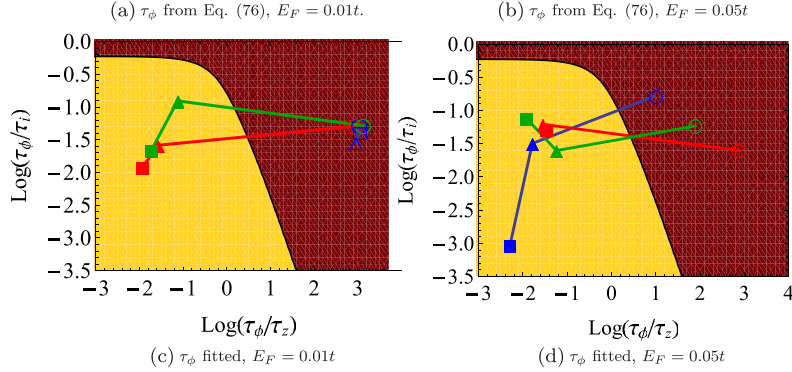

FIG. 15. (Color online) $\tau_{\phi} / \tau_{i}-\tau_{\phi} / \tau_{z}$ diagram obtained from fitting of numerical results. Empty symbols: weak localization. Filled symbols: weak antilocalization. Black line: obtained from $\Delta \sigma(B)=$ 0 in Eq. (30). 
parameter does not change the result significantly. The result of the fitting procedure for some parameter values is displayed in Fig. 14(b). As in Sec. VIII A 1, we display the resulting $1 / \tau_{i}, 1 / \tau_{z}$, and $1 / \tau_{\phi}$ in a $\tau_{\phi} / \tau_{i}-\tau_{\phi} / \tau_{z}$ diagram in Figs. $15(\mathrm{c})$ and $15(\mathrm{~d})$.

We find that the results fall in the range $\log \left(\tau_{\phi} / \tau_{i}\right) \approx$ $-3, \ldots, 0$ and $\log \left(\tau_{\phi} / \tau_{z}\right) \approx-3, \ldots, 3$. The agreement with the analytical results, i.e., the black solid line in Fig. 15, is improved. All numerical results fall on the expected side of the weak-antilocalization to weak-localization transition line.

\section{B. Ab initio calculation}

In this section, we will attempt an ab initio calculation in the sense that we use the input parameters of the numerical calculations to calculate directly the different scattering rates $1 / \tau_{i j}$, insert them into the analytical formula of Eq. (30) for the weak-localization corrections, and compare the latter to the numerical results. For this calculation, we choose the following parameter ranges. The impurity width $\xi$ is varied from $0.05 a_{0}$ to $1.5 a_{0}$ in steps of $0.05 a_{0}$, the impurity strength $V$ is varied from $0.5 \mathrm{eV}$ to $5 \mathrm{eV}$ in steps of $0.5 \mathrm{eV}$ (corresponding to $K_{0}$ between approximately 0.05 and 2) and $E_{F}$ from $0.01 t$ to $0.1 t$ in steps of $0.01 t$ (note that the analytical result is not valid too close to the Dirac point $E_{F}=0$ ). The impurity concentration is set to $3 \%$ as before, if not explicitly mentioned otherwise.

\section{Conductance}

a. Magnetoconductance sign transition. Using the explicit relations between the scattering rates and the parameters $\xi$, $V$, and $E_{F}$, as derived in Sec. VIB, in Fig. 16 we show a $\tau_{\phi} / \tau_{i}-\tau_{\phi} / \tau_{*}$ diagram

In Fig. 16, we display the magnetoconductance trajectories in the $\tau_{\phi} / \tau_{i}-\tau_{\phi} / \tau_{*}$ plane by changing the impurity parameters and $E_{F}$. For instance, this is done by changing $\xi$ for fixed $E_{F}=0.01 t$ and $V=5 \mathrm{eV}$ (red dots) and by changing $V$ and $E_{F}$ (blue/green dots), respectively, for fixed $\xi=0.05 a_{0}$ and $\xi=1.5 a_{0}$. The impurity concentration is kept fixed, with its dependence inferred from Eqs. (78) and (79).

As expected, increasing the range $\xi$ of the impurities induces a change from weak localization to weak antilocalization; see the red arrow. When increasing the impurity strength $V$, one moves toward weak localization; see the blue arrow. Increasing the Fermi energy, one also moves towards weak localization; see the green arrow. The diagram shows that $\tau_{\phi} / \tau_{*}$ saturates at fixed Fermi energy $E_{F}$ toward a lower limit since the ratio of the warping rate $1 / \tau_{w}$ and the dephasing rate $\tau_{\phi} / \tau_{w}$ converges to a finite value, given by Eq. (80), independently of $V$ and $\xi$. At small correlation lengths $\left(\xi \ll a_{0}\right)$, we find that the $1 / \tau_{z}$ term dominates; a $V^{4}$ dependence on the strength of the impurities and a $E_{F}$ dependence on the Fermi energy emerge, in agreement with Eq. (79). In the regime of large correlation lengths $\left(\xi \gg a_{0}\right)$, we observe instead a $E_{F}^{4}$ dependence, which is in agreement with the dependence of the warping rate of Eq. (80). For comparison, we plot the $\tau_{\phi} / \tau_{i}-\tau_{\phi} / \tau_{z}$ diagram without the warping term in Appendix B (Fig. 26). Next, we transform Fig. 16 into the form of the phase diagram of Fig. 7(a). The resulting $\xi-V$ magnetoconductance sign diagram is shown in Fig. 17 for two different Fermi energies.

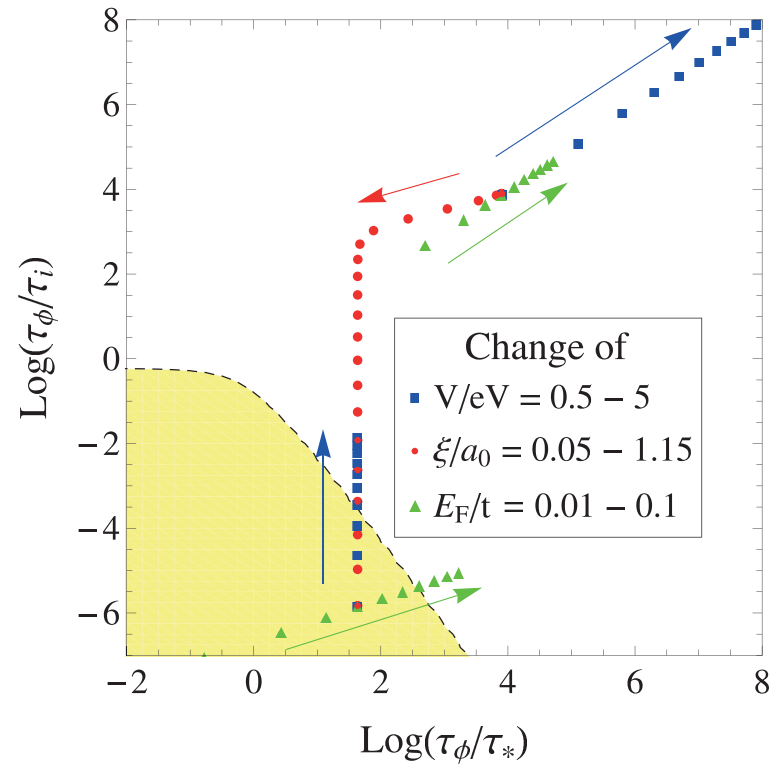

FIG. 16. (Color online) Magnetoconductance in the $\tau_{\phi} / \tau_{i}-\tau_{\phi} / \tau_{*}$ diagram. The ratios of scattering rates and dephasing rates $\tau_{\phi} / \tau_{i}$, $\tau_{\phi} / \tau_{*}$ are calculated from the parameters $\xi$ (red dots), $V$ (blue squares), and $E_{F}$ (green triangles), which are varied in the range given in the inset. Arrows indicate the direction of increasing parameter. The variation with $V$ and $E_{F}$ is shown for $\xi / a_{0}=0.05$ and $\xi / a_{0}=1.15$; $\xi$ is varied at fixed $V / \mathrm{eV}=0.5$ and $E_{F} / t=0.04$. Dashed line: localization transition. White area: PMC. Yellow area: NMC.

b. Magnetoconductance amplitude. Having investigated the dependence of the sign of the magnetoconductivity, let us now study its amplitude using Eq. (84) to evaluate Eq. (25), with $F_{\text {full }}$ given by Eq. (26).

The resulting analytical magnetoconductance amplitude is shown in Figs. 18 and 19. In Fig. 18(a), the amplitude shown in Fig. 18(b) is displayed as a function of $\tau_{\phi} / \tau_{i}$ and $\tau_{\phi} / \tau_{*}$. Since the warping term dominates for large correlation lengths $\left(\xi>a_{0}\right)$ and $\tau_{\phi} / \tau_{W}$ does not depend on $\xi$ and $V$, regions I and II collapse onto a line in Fig. 18(b). We can recognize the localization transition that we observed in the sign diagrams of

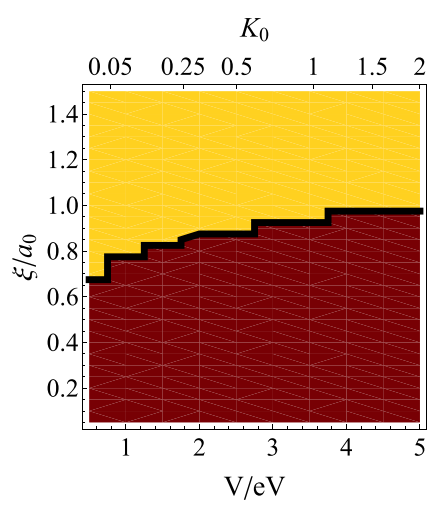

(a) $E_{F}=0.01 t$

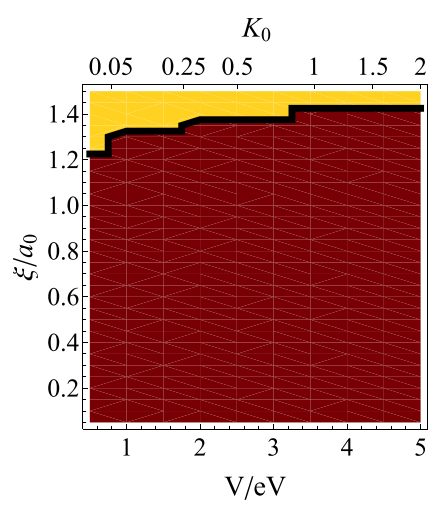

(b) $E_{F}=0.1 t$
FIG. 17. (Color online) Sign of MC as a function of $V$ and $\xi$, corresponding to Fig. 16 for two different Fermi energies. Red is PMC; yellow is NMC. Compare with the numerical results of Fig. 7(a). Black line: transition between positive and negative MC. 


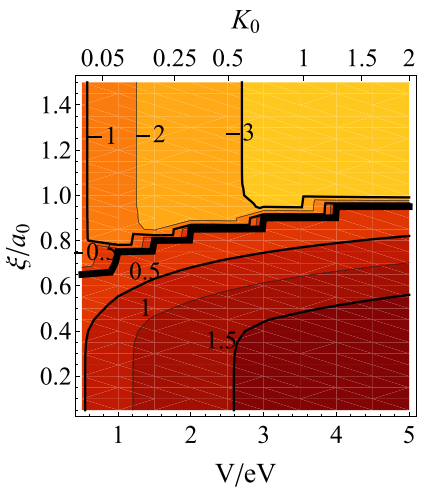

(a) $E_{F}=0.01 t$

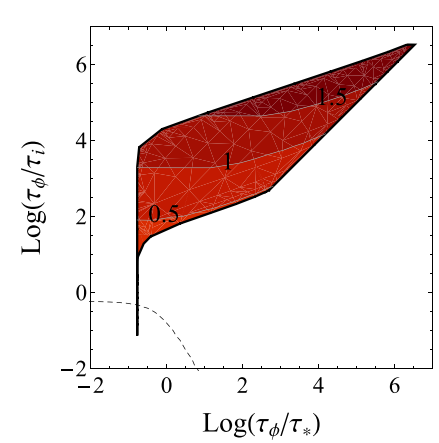

(b) $E_{F}=0.01 t$
FIG. 18. (Color online) (a) MC amplitude analytically calculated, given by Eq. (84), in units of $2 e^{2} / h$. Black line: transition between PMC and NMC. Positive numbers and red color: weak localization. (b) MC amplitude as a function of $\tau_{\phi} / \tau_{i}$ and $\tau_{\phi} / \tau_{*}$.

Fig. 17. In addition, we see that in the range of the localization transition obtained by the numerical calculation in Fig. 8, we observe weak antilocalization with a small amplitude when close to the Dirac point for small back Fermi energies and for weak scatterers (small $V, K_{0}$ ). A small weak-localization amplitude is suppressed already by a weak magnetic field. Thus, in the numerical calculations, it is difficult to identify a region with a weak-localization amplitude. We typically find, by comparison, that the low-energy cutoff in the numerical calculation is larger than the one obtained from the Thouless energy, given by Eq. (76).

\section{Quantum correction to conductance at $B=0$}

The quantum correction to the conductance shown in Eq. (23) is plotted in Fig. 20 as a function of impurity strength $V$ (and, correspondingly, $K_{0}$ ) and the correlation length $\xi$. We note that the transition line between a positive and a negative

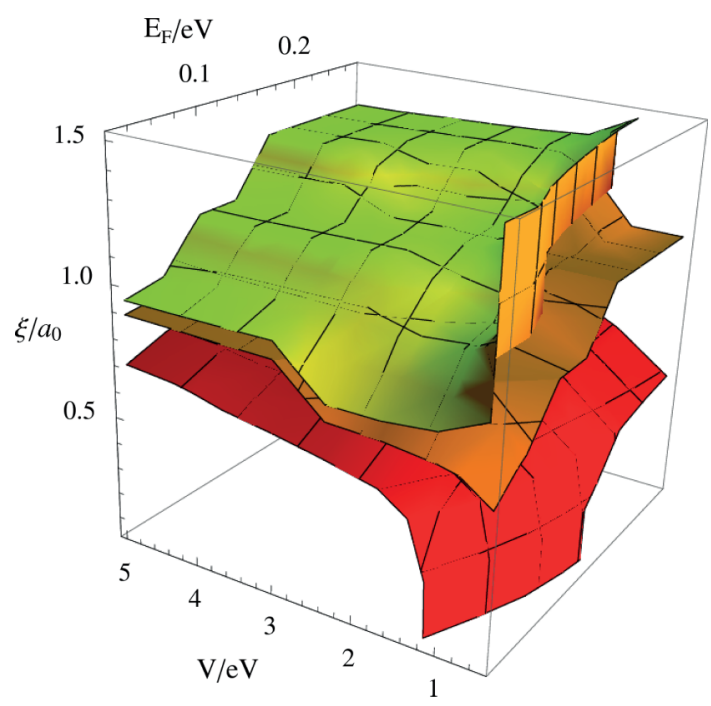

FIG. 19. (Color online) Analytically calculated magnetoconductance amplitude, given by Eq. (84), as a function of $\xi, V$, and $E_{F}$. Surfaces with value -1 (red), 0 (orange), and 1 (yellow) in units of $2 e^{2} / h$ are displayed. Grid lines are guides to the eye. quantum correction at $B=0$, as indicated by the dashed line, does not coincide with the transition from negative to positive magnetoconductance, which is indicated by the thick black line. Therefore, there is a region, denoted as II, where the quantum correction to the conductance is positive but the conductance increases with magnetic field, as one would expect for weak localization. Only in region I does the positive quantum correction coincide with the NMC expected for weak antilocalization. In region III, the negative quantum corrections yields PMC, as expected for weak localization. These regions can be related to the different types of magnetoconductance sketched in Fig. 6: applying a magnetic field in the region II, we expect a nonmonotonic magnetoconductance, where the conductance first increases, reaching a maximum at $B=B_{\mathrm{ex}}$, and then decays toward the classical conductance, as in case (2) of Fig. 6. In the other regions, I and III, the MC is monotonic, with $\mathrm{PMC}$ in region III and NMC in region I.

In Fig. 20(c), the result for the quantum correction shown in Fig. 20(a) is displayed as a function of $\tau_{\phi} / \tau_{i}$ and $\tau_{\phi} / \tau_{*}$. Since the warping term is dominant for large correlation lengths $\xi>a_{0}$, and $\tau_{\phi} / \tau_{W}$ does not depend on $\xi$ and $V$, the regions I and II collapse onto a line in Fig. 20(c).

\section{Quantum corrections to thermopower}

In this section, we use the analytical theory to study the quantum corrections to the thermopower and the resulting magnetic field dependence. In particular, we find out in which regime these corrections are large and whether, in graphene, they are dominated by the quantum corrections to the Fermi energy slope of the conductance rather than by the weak-localization corrections to the conductance, as in standard metals.

\section{Quantum corrections at zero magnetic field}

We first consider the amplitude of the quantum corrections to the thermopower as defined by Eq. (86), namely, as the difference between the value at zero magnetic field and the classical thermopower. Thus we need to use the weaklocalization correction to the conductance at zero magnetic field, $\delta \sigma(B=0)$ [see Eqs. (23) and (25)], and insert it into the Mott formula.

By inserting the dependence of the scattering rates on the Fermi energy, as given by Eqs. (72)-(74) and (76), we obtain the slope of the Fermi energy dependence of the conductance at $B=0$ as

$$
\frac{\partial \delta \sigma(B=0)}{\partial E_{F}}=\frac{e^{2}}{\pi h} \frac{1}{E_{F}} \vartheta\left(t_{i}, t_{z}, t_{w}\right),
$$

where

$$
\vartheta\left(t_{i}, t_{z}, t_{w}\right)=-2+\frac{2}{1+2 t_{i}}+\frac{4\left(1-t_{w}\right)}{1+t_{i}+t_{z}+t_{w}},
$$

which depends on the parameter ratios $t_{i}=\tau_{\phi} / \tau_{i}$ and $t_{z}=$ $\tau_{\phi} / \tau_{z}$, as well as explicitly on the warping rate ratio $t_{w}=$ $\tau_{\phi} / \tau_{w}$.

We note that while the quantum corrections to the conductivity are diverging, in the limit of $1 / \tau_{\phi} \rightarrow 0$, which corresponds to low temperatures and large system sizes, the quantum corrections to the slope of the Fermi energy 


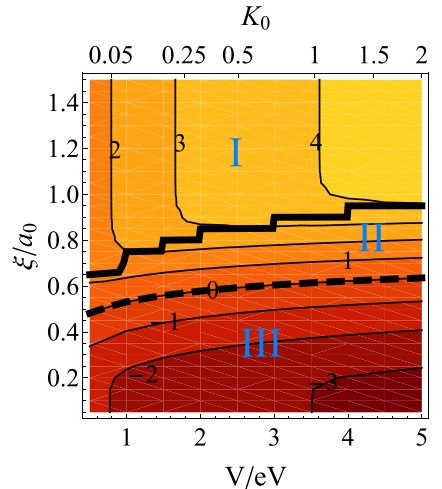

(a) $E_{F}=0.01 t$

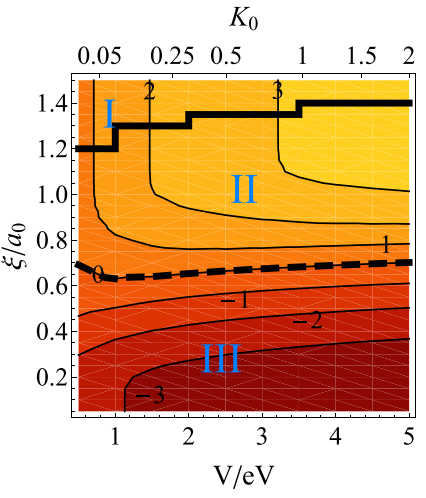

(b) $E_{F}=0.1 t$

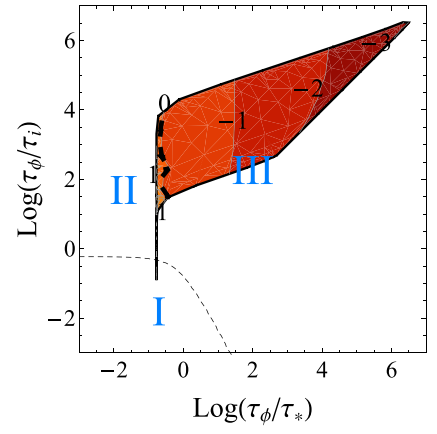

(c) $E_{F}=0.01 t$

FIG. 20. (Color online) Analytically calculated quantum correction to the conductance at zero magnetic field, $\delta \sigma(B=0)$ in units of $2 e^{2} / h$ for two different Fermi energies, (a) $E_{F}=0.01 t$ and (b) $E_{F}=0.1 t$. Black dashed line: transition from positive to negative quantum correction, $\delta \sigma(B=0)=0$. Black line: Transition from PMC to NMC. (c) Same as (a), but displayed as a function of $\tau_{\phi} / \tau_{i}$ and $\tau_{\phi} / \tau_{*}$.

dependence of the conductance, given by Eq. (87), converge to a finite value of order $\frac{e^{2}}{\pi h} \frac{1}{E_{F}}$, which depends on the scatterings rates as follows:

(1) When the warping term is negligible, $t_{w} \approx 0$, the function $\vartheta$ converges to -2 for large $t_{i}$, i.e., the weaklocalization regime, as seen in Fig. 21(a). In the weakantilocalization regime of small intervalley scattering $\left(t_{i} \ll 1\right)$, $\vartheta$ turns positive.

(2) When the warping term dominates, and $t_{z} \rightarrow 0$, the function $\vartheta$ is negative and converges for large intervalley scattering $t_{i} \gg 1$, i.e., the weak-localization regime, to -6 , as seen in Fig. 21(b). Remarkably, $\vartheta$ (and, thereby, $d \delta \sigma / d E_{F}$ ) remains for $t_{z} \rightarrow 0$ negative for all values of $t_{i}$, even in the regime of weak antilocalization.

In Fig. 22, $\vartheta$ is plotted as a function of $\xi$ and $V$. For small Fermi energy, $E_{F}=0.01 t, \vartheta$ is positive in the whole region of weak antilocalization [corresponding to phase I in Figs. 20(a) and 20(b)]. This positive enhancement of $\vartheta$ [and therefore positive quantum correction to the slope of the Fermi energy dependence of the conductance at $B=0$, given by Eq. (87)] matches well with the numerical results of Sec. VII B. In that regime, the intravalley scattering rate $1 / \tau_{z}$ is expected to dominate over the warping rate, and the values of $\vartheta$ indeed agree with those obtained in Fig. 21(a) where $t_{w}=0$. At higher
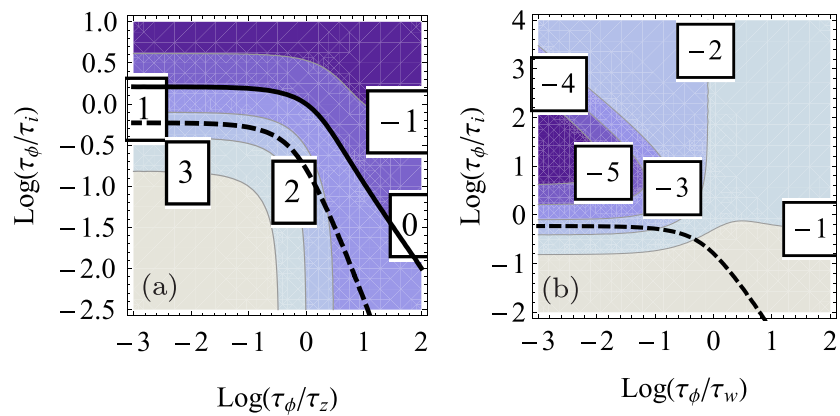

FIG. 21. (Color online) The function $\vartheta$, given by Eq. (88), (a) for $\tau_{\phi} / \tau_{w}=0$ and (b) for $\tau_{\phi} / \tau_{z}=0$. The continuous black line indicates the sign change of the function $\vartheta$. Dashed line: transition between $\mathrm{PMC}$ and NMC.
Fermi energies, shown in Fig. 22(b), the warping rate becomes more important, as $t_{W} \sim E_{F}^{4}$ increases faster than $t_{z}$ with $E_{F}$. Indeed, $\vartheta$ remains negative for all values of $\xi$ and $V$, in agreement with Fig. 21(b), where the intralayer scattering rate is set to zero, $t_{z}=0$. In the regime I of weak antilocalization, only a slight increase of $\vartheta$ is seen, while it remains negative.

Now we are in a position to consider the quantum corrections to the thermopower $\delta S$ as given in Eq. (85). For small Fermi energies, these corrections are dominated by the second term in Eq. (85), resulting in the weak-antilocalization regime (I) in a negative correction $\delta S / T<0$ of order $\frac{\pi^{2}}{3} \frac{k_{B}^{2}}{|e| e V}=$ $0.024 \mu \mathrm{V} / \mathrm{K}^{2}$. In the regime of weak localization, the quantum correction to the thermopower becomes positive. Since the classical magnetoconductance increases with gate voltage, we find that the first term in Eq. (85) becomes dominant at large Fermi energies, and one recovers $\delta S / S_{\mathrm{cl}} \approx-\delta G / G_{\mathrm{cl}}$, which is characteristic of standard metals. To study the competition between these two terms in more detail, we need an expression for the classical conductivity. In the strong scattering limit of Gaussian impurities and for Coulomb scatterers, one obtains a

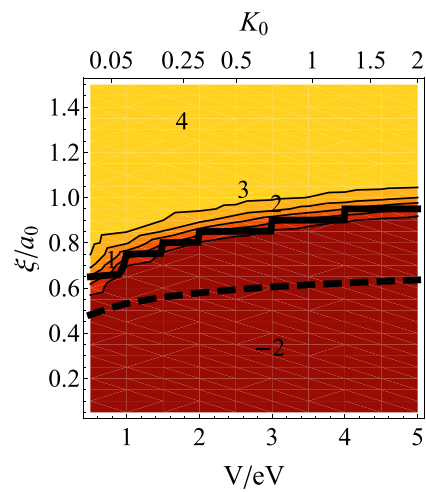

(a) $E_{F}=0.01 t$

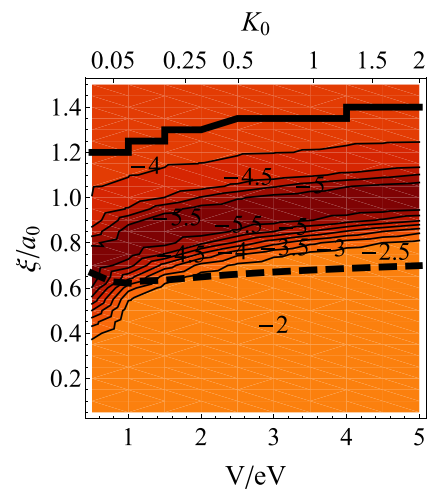

(b) $E_{F}=0.1 t$
FIG. 22. (Color online) $\vartheta$, given by Eq. (88), for two different Fermi energies as a function of $\xi$ and $V$. As in Figs. 20(a) and 20(b), the black dashed line indicates the transition from positive to negative quantum correction to the conductance, $\delta \sigma(B=0)=0$. Black line: transition from PMC to NMC. 


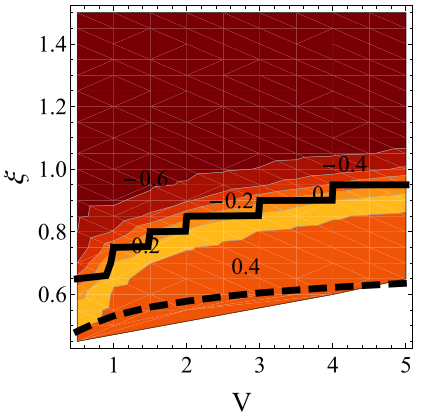

(a) $E_{F}=0.01 t$

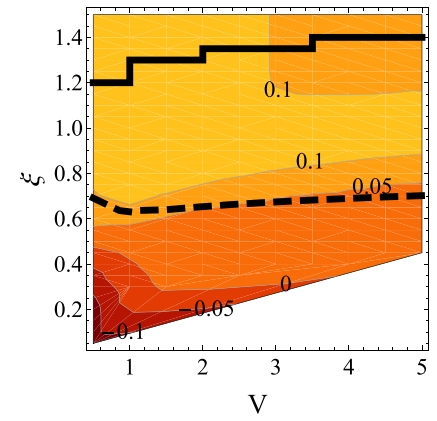

(b) $E_{F}=0.1 t$
FIG. 23. (Color online) Analytically calculated $\delta S / T$ obtained from Eq. (90) in units of $\mu \mathrm{V} / \mathrm{K}^{2}$ for two different Fermi energies. Black line: transition from PMC to NMC. Dashed line: $\delta \sigma=0$. White area: weak-localization correction to conductance exceeds classical value.

quadratic dependence on Fermi energy [17],

$$
\sigma_{\mathrm{cl}}=\frac{4 e^{2}}{\pi h}+c_{\xi} \frac{2 e^{2} E_{F}^{2}}{h V^{2}},
$$

which is well justified in the limit of strong scatterers [13]. The prefactor $c_{\xi}$ is of the order of unity and increases from short-range to long-range scatterers by a factor of 2 [13]. Note that we use here the notation introduced in Sec. VI, where $V$ is a measure of the total impurity strength averaged over all impurities and increases with the density of impurities $n_{\text {imp }}$ as $V \sim \sqrt{n_{\text {imp }}}$. We then obtain $S_{\text {cl }}$ by inserting Eq. (89) into the Mott formula, given by Eq. (31). We obtain $S_{c l}$ by inserting Eq. (89) into the Mott formula, given by Eq. (31). With Eq. (24) for $\delta \sigma$ and Eq. (87) for $\partial \delta \sigma / \partial E_{F}$, we can use Eq. (85) to find the dependence of $\delta S / T$ on the impurity parameter and the Fermi energy. We obtain

$$
\frac{\delta S}{T}=\frac{\pi^{2} k_{B}^{2} V^{2}}{3|e|\left(2 V^{2}+\pi E_{F}^{2}\right)}\left(\frac{\pi E_{F}}{2 V^{2}+\pi E_{F}^{2}} \theta-\frac{1}{2 E_{F}} \vartheta\right) .
$$

This result is displayed in Fig. 23, where we set $c_{\xi}=1$. The white area corresponds to the regime where $\sigma_{\text {Diff }} \ll \Delta \sigma(B=$ 0 ), where Eq. (85) is no longer valid. [We note that, more generally, one needs to take into account that the classical conductivity given by Eq. (89) depends also on the range of impurities, and for weak scatterers it may attain a weaker logarithmic energy dependence [13]. This will change these results quantitatively, but is not expected to change them qualitatively, which is why we choose Eq. (89) and leave the inclusion of a more consistent quantitative analysis for the classical conductivity for future studies.]

Comparing the results with Fig. 22, we can see that the term due to the quantum corrections to the Fermi energy slope of the conductance, i.e., the $\vartheta$ term in Eq. (90), is dominant in our parameter range. The quantum corrections of the conductance, i.e., the $\theta$ term, play a minor role here. However, that term gains importance with higher Fermi energy. For small Fermi energy, the intravalley scattering $1 / \tau_{z}$ dominance is visible in phase I weak antilocalization (WAL), where we detect a negative amplitude of the correction $\delta S / T$, while in phase II and III, the correction is positive. For higher Fermi energy, the warping term $1 / \tau_{w}$ is becoming dominant and we can observe a positive correction for the complete phase I and II, and even a small correction for parts of phase III.

To see the connection between the electrical conductivity correction and the thermopower correction, we plot $\delta \sigma$ versus $\delta S / T$ in Fig. 24. We focus on the range of weak antilocalization (phases I and II). For small Fermi energy, shown in Fig. 24(a), we can see the transition from positive to negative thermopower correction $\delta S$ when increasing the impurity size $\xi$. We find negative $\delta S<0$ in phase I, while positive $\delta S>0$ is seen in phase II. An increase of the impurity strength is moving the system toward positive $\delta S>0$. This behavior changes at higher Fermi energy [see Fig. 24(b)], since the warping term becomes more important. For short-range impurities, the system in phase II does not show a clear relation between $\delta \sigma$ and $\delta S / T$. An increase of the impurity strength tends to lower the thermopower correction. With increasing impurity range, when $\xi \approx a_{0}$, we observe an increase of the thermopower correction with an increase of the conductivity correction near the transition from NMC to PMC. For longerranged impurities, the system is in phase I of NMC. In that regime, we find good agreement with the numerical results and observe a clear relation between an increase of $\delta S / T$ and $\delta \sigma$. We note that the detailed parameter dependence may vary, depending on the value of the classical conductance $\sigma_{\text {Diff }}$ and the low-energy cutoff $1 / \tau_{\phi}$.

\section{Magnetothermopower}

Next, we consider how these quantum corrections change when applying a magnetic field. We focus first on the derivative

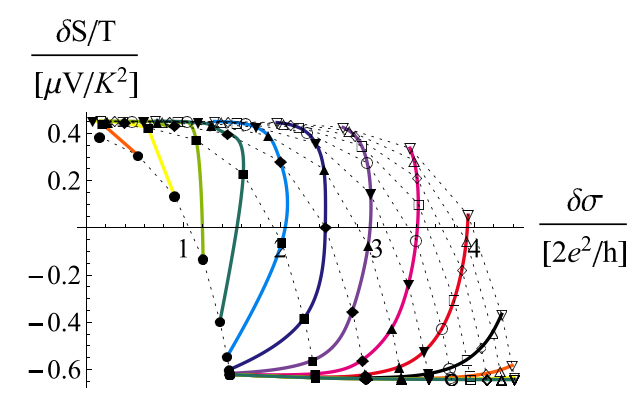

(a) $E_{F}=0.01 t$

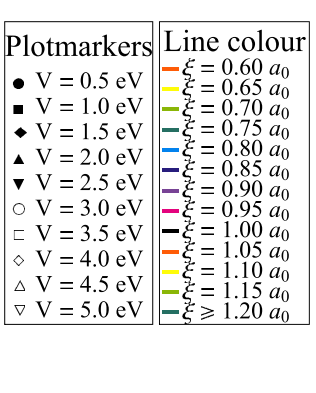

(b) $E_{F}=0.1 t$

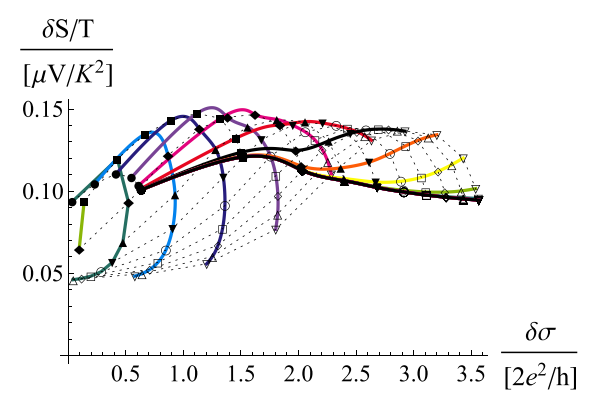

FIG. 24. (Color online) Analytically calculated $\delta S / T-\delta \sigma$ diagram at zero magnetic field displaying the relation between the quantum corrections to thermopower and conductivity corrections for the phases of WAL, I and II, for various impurity parameters $\xi$ and $V$, for two different Fermi energies. Dotted black lines: same impurity strength for different $\xi$. 


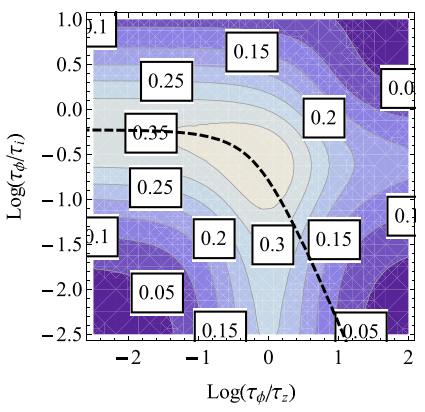

(a) $\kappa\left(t_{i}, t_{z}, t_{w}\right)$ in a $\tau_{\phi} / \tau_{z}$ $\tau_{\phi} / \tau_{i}$ diagram for $1 / \tau_{w}=0$

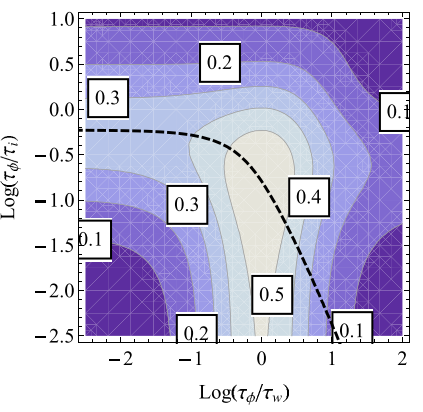

(b) $\kappa\left(t_{i}, t_{z}, t_{w}\right)$ in a $\tau_{\phi} / \tau_{w}$ $\tau_{\phi} / \tau_{i}$ diagram for $1 / \tau_{z}=0$.
FIG. 25. (Color online) $\kappa\left(t_{i}, t_{z}, t_{w}\right)$ displayed for the two limiting cases $1 / \tau_{w}=0$ and $1 / \tau_{z}=0$. Dashed lines: NMC to PMC transition from Fig. 1. Positive numbers indicate positive magnetothermopower (PMT), an increase of $\delta S / T$ for small magnetic fields.

with respect to the Fermi energy. To this end, we can use the expansion at weak magnetic fields for $\Delta \sigma(B)$, given by Eq. (30), and take its derivative with respect to the Fermi energy $E_{F}$. For the finite-size samples used in the numerical calculations, we can substitute $1 / \tau_{\phi}=E_{c}=D / \Lambda^{2}$ and find that the prefactor in Eq. (30) does not depend on $E_{F}$. Thus, we find

$$
\Delta \frac{d \sigma(B)}{d E_{F}} \approx \frac{e^{2}}{24 \pi h}\left(\frac{4 e B \Lambda^{2}}{\hbar}\right)^{2} \frac{4}{E_{F}} \kappa\left(t_{i}, t_{z}, t_{w}\right),
$$

where

$$
\kappa\left(t_{i}, t_{z}, t_{w}\right)=\left[\frac{2 t_{i}}{\left(1+2 t_{i}\right)^{3}}+\frac{t_{i}+t_{z}+2 t_{w}}{\left(1+t_{i}+t_{z}+t_{w}\right)^{2}}\right] .
$$

This is a purely positive magnetothermopower whose amplitude increases with system size. We display $\kappa\left(t_{i}, t_{z}, t_{w}\right)$ in the limits of $1 / \tau_{w}=0$ [Fig. 25(a)] and $1 / \tau_{z}=0$ [Fig. 25(b)].

For higher magnetic fields, we can use the full expression and find

$$
\frac{\partial \Delta \sigma(B)}{\partial E_{F}}=\frac{e^{2}}{\pi h} \frac{4}{E_{F}} \varsigma\left(\tau_{i}, \tau_{z}, \tau_{w}, \tau_{\phi}, \tau_{B}\right),
$$

where

$$
\begin{aligned}
& \varsigma\left(\tau_{i}, \tau_{z}, \tau_{w}, \tau_{\phi}, \tau_{B}\right) \\
&=\left\{\frac{\tau_{B}}{2 \tau_{0}} \Psi_{1}\left(\frac{1}{2}+\frac{\tau_{B}}{2 \tau_{0}}\right)-\frac{\tau_{B}}{\tau_{i}} \Psi_{1}\left[\frac{1}{2}+\tau_{B}\left(\frac{2}{\tau_{i}}+\frac{1}{\tau_{\phi}}\right)\right]\right. \\
&-\tau_{B}\left(\frac{1}{\tau_{i}}+\frac{2}{\tau_{w}}+\frac{1}{\tau_{z}}\right) \Psi_{1}\left[\frac{1}{2}+\tau_{B}\left(\frac{1}{\tau_{w}}+\frac{1}{\tau_{i}}\right.\right. \\
&\left.\left.\left.+\frac{1}{\tau_{z}}+\frac{1}{\tau_{\phi}}\right)\right]\right\},
\end{aligned}
$$

and $\Psi_{1}$ is the polygamma function $\Psi_{n}$ with $n=1$, also known as the trigamma function. Performing the derivative shows that the pseudospin-singlet isospin-singlet term does not contribute. When the magnetic field reaches $B_{\max }$, the magnetothermopower amplitude vanishes, as does the magnetoconductivity. We find that the thermopower correction $\delta S / T$ has a peak for higher magnetic fields when the value for $B=0$ is positive. This is in accordance with our numerical finding; see Fig. 12.

\section{ANALYSIS OF EXPERIMENTAL RESULTS}

There have been several reports on magnetoconductance experiments on single-layer graphene. The weak-localization amplitude is typically of the order of $2 e^{2} / h[2,3,33-36]$. Tikhonenko and co-workers showed that it is possible to observe weak localization up to a temperature of $200 \mathrm{~K}$ [3]. In their work, they found a clear transition between PMC and NMC as the temperature is changed. An increase of the temperature decreases the MC amplitude due to the increasing of the dephasing rate, $1 / \tau_{\phi}(T)$. For example, a change from 5 to 14 Kelvin in Ref. [3] reduces the amplitude at gate voltage $V_{G}=40 \mathrm{~V}$ by $70 \%$. For this reason, to maximize the amplitude, most experiments are done at temperatures below $10 \mathrm{~K}$. The MC effect is visible in all experiments at magnetic fields of up to $B=0.1 T$. In addition, in all experiments, there are observable magnetoconductance fluctuations whose amplitudes are, however, much smaller than the total MC amplitude.

We focus on Ref. [3] at the lowest temperature, $T=5 \mathrm{~K}$, since it has the largest amplitude. The scattering rates reported by the authors are obtained by fitting the analytical theory of Ref. [4]. They are found to be $\log \left[\tau_{\phi} / \tau_{*}\right]=1.3,2,2.4$ and $\log \left[\tau_{\phi} / \tau_{i}\right]=-0.2,1.17,2$ for the three gate voltages $V_{G}=$ $7,20,40 \mathrm{~V}$.

The Mott formula, given by Eq. (31), can also be written in terms of the back gate voltage $V_{\mathrm{BG}}$ as

$$
S=\left.\frac{\pi^{2}}{3} \frac{k_{B}^{2} T}{e} \frac{1}{\sigma} \frac{d \sigma}{d V_{\mathrm{BG}}} \frac{d V_{\mathrm{BG}}}{d E}\right|_{E=E_{F}},
$$

with

$$
E_{F}=\hbar v_{F} \sqrt{\pi n_{2 \mathrm{D}}} \propto \pm \sqrt{\left|V_{\mathrm{BG}}\right|}
$$

where $n_{2 \mathrm{D}}$ is the two-dimensional carrier density.

Using the dephasing rate $1 / \tau_{\phi}=0.1 \mathrm{ps}^{-1}$ as measured in Ref. [3] at $T=5 \mathrm{~K}$, identifying $V_{G}=V_{\mathrm{BG}}$, and taking for the impurity concentration $3 \%$, we find a very good agreement between experiments and our results shown in Fig. 16. We find the same values for the scattering rate ratios $\tau_{\phi} / \tau_{*}$ and $\tau_{\phi} / \tau_{i}$ if we set the impurity parameters to $\xi \lesssim 0.5 a_{0}$ and $V \approx 0.5 \mathrm{eV}$ in the $a b$ initio calculations outlined above. Thus, we conclude that the analysis of the experimental results with the theory allows us to make detailed predictions about the potential amplitude and range of the impurities in the graphene samples. For the sample of Ref. [3], which was produced by mechanical exfoliation of graphite and deposition on an oxidized Si wafer, we can conclude that the typical range of the impurities in that sample is $\xi \lesssim 0.5 a_{0}$. If the impurity concentration were known from an independent measurement, their average strength $V$ could be inferred from these magnetoconductance measurements as well. By using the measured mobility of $\mu_{e}=12000 \mathrm{~cm}^{2} /(\mathrm{V} \mathrm{s})$, one could, in principle, estimate one of these parameters.

Comparing with the analytical results, shown in Fig. 16, we see that the experimental results for gate voltages $V_{G}=7 \mathrm{~V}$ and $V_{G}=20 \mathrm{~V}$ are in the regime where the intravalley scattering rate $1 / \tau_{z}$ is dominant, while at a gate voltage of $40 \mathrm{~V}$, this rate is superseded by the warping rate $1 / \tau_{w}$. A higher accuracy of the data analysis can be achieved by fitting 
the experimental data at different gate voltages to the same parameters and making use of the analytical gate voltage dependence of $\tau_{\phi} / \tau_{i}$ and $\tau_{\phi} / \tau_{*}$, as in Eq. (80).

There have been several reports on the measurements of thermopower in single-layer graphene [5-8]. We found above that the magnitude of the quantum corrections to thermopower is of the order of $1 \mu \mathrm{V} / T^{2}$ [see Figs. 13(a) and 24], which is not much smaller than the measured values for the classical thermopower of single-layer graphene [6-8]. We note that the dependence of $\delta \sigma$ on $E_{F}$ strongly depends on the Fermi energy dependence of the dephasing rate $1 / \tau_{\phi}$. At low temperatures, the dephasing is dominated by the electron-electron scattering, yielding [37]

$$
\frac{1}{\tau_{\phi}}=\alpha \frac{k_{B} T}{2 E_{F} \tau_{0}} \ln \left(2 E_{F} \tau_{0} / \hbar\right) .
$$

Since $1 / \tau_{0} \sim E_{F}$, given by Eq. (74), close to the Dirac point, one finds that $\tau_{\phi}$ is independent of the Fermi energy $E_{F}$, in good agreement with the experimental results [3].

High magnetic field thermopower measurements at room temperature have been performed [38] and a theory based on the self-consistent Born approximation (SCBA) has been given in Ref. [39]. The temperature scale of these experiments ranges from room temperature down to several Kelvin, which would also be the right temperature range to observe the magnetothermopower due to quantum corrections studied here. To this end, the thermopower measurements would have to be performed at magnetic fields below $B_{\max } \sim 0.1$ Tesla. We are not aware that such measurements have been performed to date.

As mentioned before, the weak-localization corrections to the conductance and thermopower become suppressed when the magnetic length $l_{B}$ is on the order of the elastic mean free path $l_{e}=v \tau$ or when $1 / \tau \sim v / l_{B}$, which defines the maximal magnetic field $B_{\max }$ at which weak-localization corrections can be expected. The spacing between the Landau levels in graphene is known to be anomalously large, $\hbar \omega_{c}=\sqrt{2} v / l_{B}$. The condition where Landau levels become smeared out by disorder yields, therefore, $1 / \tau \sim v / l_{B}$, and in the weak-localization regime, $B<B_{\max }$ effects of Landau bands can indeed be disregarded. For $B>B_{\max }$, the magnetoconductance is classical, but can also be sensitive to the range of impurities and acquire a $\sqrt{B}$ dependence $[40,41]$. Thus, the magnetothermopower accordingly can be expected to become stronger than previously known [39].

There is increasing evidence that adatoms and vacancies are important to understand the transport properties of graphene. The transport theory of graphene with such strong impurities forming resonances has recently been studied in Ref. [42], where it was found that there is a regime close to the Dirac point where the transport differs from the predictions based on the transport theory with nonresonant impurities used here. It will therefore be important to study the thermopower in the presence of such impurities. However, Ref. [42] confirms that there is a regime away from the Dirac point where the effect of strong impurities can be described by the model used here.

\section{CONCLUSIONS AND DISCUSSION}

We have studied the quantum corrections to the conductivity and the thermopower in monolayer graphene by analyzing numerical results in conjunction with the analytical theory. The quantum corrections to the thermopower result in large magnetothermopower, which we demonstrate to be a very sensitive measure of the impurities in graphene. While there are experimental measurements of magnetoconductance of single-layer graphene which could be used to determine the average range and strength of the impurities, future measurements of the magnetothermopower could provide additional information about the graphene samples. The strong magnetic field dependence of the thermopower, i.e., the magnetothermopower, is a direct measure of the quantum corrections to the thermopower. In contrast to usual metals, its amplitude in graphene is not simply related to the amplitude of the electrical conductivity, but is also governed by the quantum correction to the gate voltage slope of the conductivity.

We demonstrate that the numerical calculations not only can be fitted with the analytical theory, but we even find good agreement when the scattering rates entering the analytical theory are calculated directly from the numerical input parameters. This allows a more detailed understanding, since the dependence on gate voltage and other variable parameters can be studied, revealing more accurate information on the impurity parameters in graphene.

Besides the sign of the magnetoconductance, showing a transition between PMC and NMC, we also analyzed the amplitude of the quantum corrections. We found that the transition from PMC to NMC does not coincide with the transition from positive to negative quantum corrections at vanishing magnetic field $B=0$. We analyzed the relation between the amplitude of the quantum corrections to the conductance and the magnetothermopower. It appeared strongest close to the Dirac point and for long-range impurities.

Here, we studied the thermopower using the Mott formula, which is only valid for small ratios $T / E_{F}$. Still, experiments show that it remains valid close to the Dirac point $[5,9]$. We intend to study its validity in the future by using directly the Kubo formula for the thermopower. For the aspect ratio 1 studied in this paper, we did not find a strong sensitivity to the form of the boundary conditions, i.e., armchair or zigzag. The quantum corrections are expected to increase for larger aspect ratios. Also the warping rate $1 / \tau_{w}$ is expected to be sensitive to the aspect ratio, being suppressed when the width of the graphene samples is reduced. This effect is similar to the reduction of the Dyakonov-Perel spin scattering rate in quantum wires [43]. The other rates $1 / \tau_{i}$ and $\tau_{z}$ are expected to depend only weakly on the aspect ratio. We note that we assumed in this paper that the dominant impurity scattering processes are elastic. The effect of magnetic scatterers on the weak-localization corrections in graphene will be studied in a separate work.

\section{ACKNOWLEDGMENTS}

The authors would like to thank Hyunyong Lee, Paul Wenk, Vincent Sacksteder, Seung-Geol Nam, and Prof. Hu Jong Lee for useful discussions. We gratefully acknowledge that this research was supported by the World Class University (WCU) program, Division of Advanced Materials Science as well as the Asian Pacific Center of Theoretical Physics (APCTP) at POSTECH university. We also thank the Jacobs University Foundation for supporting this research. 


\section{APPENDIX A: MATRIX NOTATION OF THE HAMILTONIAN}

For a better understanding of the structure of our formulation, it useful to write the Hamiltonian in matrix notation. We present here $H_{1}, H_{2}$, and $H_{\text {imp }}$ in this way. In particular, the $H_{\text {imp }}$ matrix makes it easier to see the connection of each part of the impurity potential to the isospin-pseudospin description and the matrix notation of the Gaussian potential which is shown in Sec. VI. We have

$$
\begin{aligned}
H_{1} & =v_{F} \zeta_{3} \otimes \vec{\sigma} \cdot \vec{k} \\
& =v_{F}\left(\begin{array}{cccc}
0 & k_{x}-i k_{y} & 0 & 0 \\
k_{x}+i k_{y} & 0 & 0 & 0 \\
0 & 0 & 0 & k_{x}+i k_{y} \\
0 & 0 & k_{x}-i k_{y} & 0
\end{array}\right),
\end{aligned}
$$

and

$$
\begin{aligned}
H_{2} & =\mu\left[\sigma_{1}\left(k_{x}^{2}-k_{y}^{2}\right)-2 \sigma_{2}\left(k_{x} k_{y}\right)\right] \\
& =\mu\left(\begin{array}{cccc}
0 & \left(k_{x}+i k_{y}\right)^{2} & 0 & 0 \\
\left(k_{x}-i k_{y}\right)^{2} & 0 & 0 & 0 \\
0 & 0 & 0 & \left(k_{x}+i k_{y}\right)^{2} \\
0 & 0 & \left(k_{x}-i k_{y}\right)^{2} & 0
\end{array}\right) .
\end{aligned}
$$

The second term of the impurity Hamiltonian, as given in Eq. (11), can be rewritten in matrix form as

$\left.\begin{array}{cc|cc}V_{3,3} & V_{1,3}-i V_{2,3} & V_{3,1}-i V_{3,2} & -V_{1,1}+i V_{1,2}+i V_{2,1}+V_{2,2} \\ V_{1,3}+i V_{2,3} & -V_{3,3} & V_{1,1}-i V_{1,2}+i V_{2,1}+V_{2,2} & V_{3,1}-i V_{3,2} \\ \hline V_{3,1}+i V_{3,1} & V_{1,1}+i V_{1,2}-i V_{2,1}+V_{2,2} & -V_{3,3} & V_{1,3}-i V_{2,3} \\ -V_{1,1}-i V_{1,2}-i V_{2,1}+V_{2,2} & V_{3,1}+i V_{3,1} & V_{1,3}+i V_{2,3}\end{array}\right)$.

APPENDIX B: MAGNETOCONDUCTANCE FOR $1 / \tau_{w} \rightarrow 0$

In Fig. 26, we present the results of the ab initio calculations for the MC as a function of $\tau_{\phi} / \tau_{i}$ and $\tau_{\phi} / \tau_{z}$.

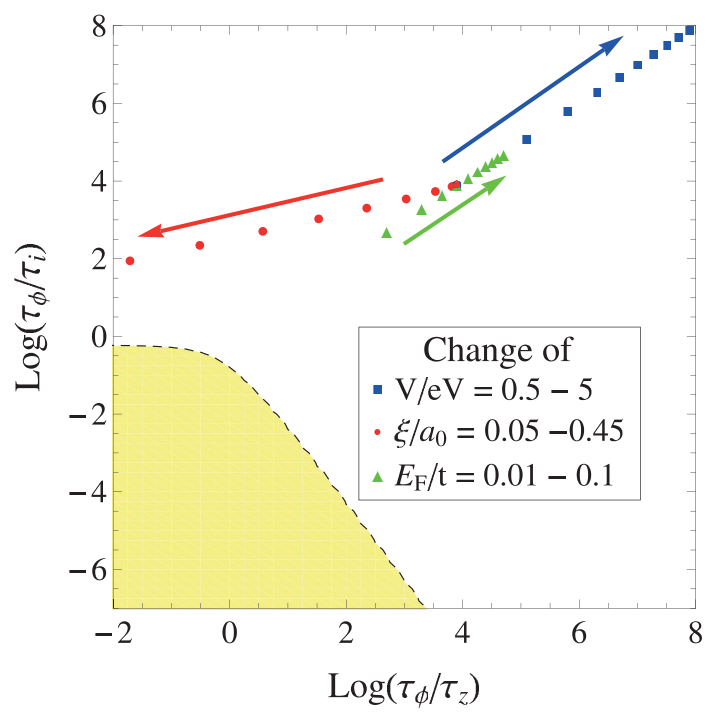

FIG. 26. (Color online) Magnetoconductance in the $\tau_{\phi} / \tau_{i}-\tau_{\phi} / \tau_{z}$ diagram for $1 / \tau_{w}=0$. The ratios of scattering rates and dephasing rates $\tau_{\phi} / \tau_{i}, \tau_{\phi} / \tau_{z}$ are calculated from the parameters $\xi$ (red dots), $V$ (blue squares), and $E_{F}$ (green triangles), which are varied in the range given in the inset. Arrows indicate the direction of increasing parameter. The variation with $V$ and $E_{F}$ is shown for $\xi / a_{0}=.05$; $\xi$ is varied at fixed $V / \mathrm{eV}=0.5$ and $E_{F} / t=.04$. Dashed line: localization transition. White area: PMC. Yellow area: NMC. 
[1] K. S. Novoselov, A. K. Geim, S. V. Morozov, D. Jiang, Y. Zhang, S. V. Dubonos, I. V. Grigorieva, and A. A. Firsov, Science 306, 666 (2004).

[2] D.-K. Ki, D. Jeong, J.-H. Choi, H.-J. Lee, and K.-S. Park, Phys. Rev. B 78, 125409 (2008).

[3] F. V. Tikhonenko, A. A. Kozikov, A. K. Savchenko, and R. V. Gorbachev, Phys. Rev. Lett. 103, 226801 (2009).

[4] E. McCann, K. Kechedzhi, V. I. Fal'ko, H. Suzuura, T. Ando, and B. L. Altshuler, Phys. Rev. Lett. 97, 146805 (2006).

[5] S.-G. Nam, D.-K. Ki, and H.-J. Lee, Phys. Rev. B 82, 245416 (2010).

[6] J. G. Checkelsky and N. P. Ong, Phys. Rev. B 80, 081413 (2009).

[7] Y. M. Zuev, W. Chang, and P. Kim, Phys. Rev. Lett. 102, 096807 (2009).

[8] P. Wei, W. Bao, Y. Pu, C. N. Lau, and J. Shi, Phys. Rev. Lett. 102, 166808 (2009).

[9] T. Löfwander and M. Fogelström, Phys. Rev. B 76, 193401 (2007).

[10] V. V. Afonin, Y. M. Gal'perin and V. L. Gurevich, Zh. Eksp. Teor. Fiz 87, 335 (1984) [Sov. Phys. JETP 60, 194 (1984)].

[11] V. V. Afonin, Y. M. Gal'perin, and V. L. Gurevich, Phys. Rev. B 33, 8841 (1986).

[12] M. J. Kearney and P. N. Butcher, J. Phys. C 21, L265 (1988).

[13] P. M. Ostrovsky, I. V. Gornyi, and A. D. Mirlin, Phys. Rev. B 74, 235443 (2006).

[14] I. L. Aleiner and K. B. Efetov, Phys. Rev. Lett. 97, 236801 (2006).

[15] S. Guruswamy, A. LeClair, and A. W. W. Ludwig, Nucl. Phys. B 583, 475 (2000).

[16] D. V. Khveshchenko, Phys. Rev. Lett. 97, 036802 (2006).

[17] A. H. Castro Neto, F. Guinea, N. M. R. Peres, K. S. Novoselov, and A. K. Geim, Rev. Mod. Phys. 81, 109 (2009).

[18] T. Ando, T. Nakanishi, and R. Saito, J. Phys. Soc. Jpn. 67, 2857 (1998).

[19] V. I. Falko, K. Kechedzhi, E. McCann, B. Altshuler, H. Suzuura, and T. Ando, Solid State Commun. 143, 33 (2007).

[20] P. A. Lee and D. S. Fisher, Phys. Rev. Lett. 47, 882 (1981).

[21] R. A. Jalabert, H. U. Baranger, and A. D. Stone, Phys. Rev. Lett. 65, 2442 (1990).
[22] E. R. Mucciolo and C. H. Lewenkopf, J. Phys.: Condens. Matter 22, 273201 (2010).

[23] E. R. Mucciolo, A. H. Castro Neto, and C. H. Lewenkopf, Phys. Rev. B 79, 075407 (2009).

[24] C. H. Lewenkopf, E. R. Mucciolo, and A. H. Castro Neto, Phys. Rev. B 77, 081410 (2008).

[25] C. Lewenkopf and E. Mucciolo, J. Comput. Electron. 12, 203 (2013).

[26] H. Schomerus, Phys. Rev. B 76, 045433 (2007).

[27] D. Ferry and S. Goodnick, Transport in Nanostructures, Cambridge Studies in Semiconductor Physics and Microelectronic Engineering (Cambridge University Press, Cambridge, UK, 1997).

[28] A. Rycerz, J. Tworzydlo, and C. W. J. Beenakker, Europhys. Lett. 79, 57003 (2007).

[29] T. Ando, J. Phys. Soc. Jpn. 74, 777 (2005).

[30] J. T. Edwards and D. J. Thouless, J. Phys. C 5, 807 (1972).

[31] P. A. Lee and T. V. Ramakrishnan, Rev. Mod. Phys. 57, 287 (1985).

[32] I. L. Aleiner and V. I. Fal'ko, Phys. Rev. Lett. 87, 256801 (2001).

[33] J. Eroms and D. Weiss, New J. Phys. 11, 095021 (2009).

[34] X. Wu, X. Li, Z. Song, C. Berger, and W. A. de Heer, Phys. Rev. Lett. 98, 136801 (2007).

[35] F. V. Tikhonenko, D. W. Horsell, R. V. Gorbachev, and A. K. Savchenko, Phys. Rev. Lett. 100, 056802 (2008).

[36] D. Horsell, F. Tikhonenko, R. Gorbachev, and A. Savchenko, Phil. Trans. R. Soc. A 366, 245 (2008).

[37] B. L. Altshuler, A. G. Aronov, and D. E. Khmelnitsky, J. Phys. C 15, 7367 (1982).

[38] X. Liu, D. Wang, P. Wei, L. Zhu, and J. Shi, Phys. Rev. B 86, 155414 (2012).

[39] B. Dóra and P. Thalmeier, Phys. Rev. B 76, 035402 (2007).

[40] P. S. Alekseev, A. P. Dmitriev, I. V. Gornyi, and V. Yu. Kachorovskii, Phys. Rev. B 87, 165432 (2013).

[41] G. Vasileva, P. Alekseev, Y. Ivanov, Y. Vasilev, D. Smirnov, H. Schmidt, R. Haug, F. Gouider, and G. Nachtwei, JETP Lett. 96, 471 (2012).

[42] G. Trambly de Laissardière and D. Mayou, Phys. Rev. Lett. 111, 146601 (2013).

[43] S. Kettemann, Phys. Rev. Lett. 98, 176808 (2007). 